\title{
Straightening Polygonal Arcs and Convexifying Polygonal Cycles
}

\author{
Robert Connelly*
}

\author{
Erik D. Demaine ${ }^{\dagger}$
}

February 7, 2002

\begin{abstract}
Consider a planar linkage, consisting of disjoint polygonal arcs and cycles of rigid bars joined at incident endpoints (polygonal chains), with the property that no cycle surrounds another arc or cycle. We prove that the linkage can be continuously moved so that the arcs become straight, the cycles become convex, and no bars cross while preserving the bar lengths. Furthermore, our motion is piecewise-differentiable, does not decrease the distance between any pair of vertices, and preserves any symmetry present in the initial configuration. In particular, this result settles the well-studied carpenter's rule conjecture.
\end{abstract}

\section{Introduction}

A planar polygonal arc or open polygonal chain is a sequence of finitely many line segments in the plane connected in a path without self-intersections. It has been an outstanding question as to whether it is possible to continuously move a polygonal arc in such a way that each edge remains a fixed length, there are no self-intersections during the motion, and at the end of the motion the arc lies on a straight line. This has come to be known as the carpenter's rule problem. A related question is whether it is possible to continuously move a polygonal simple closed curve in the plane, often called a closed polygonal chain or polygon, again without creating self-intersections or changing the lengths of the edges, so that it ends up a convex closed curve (see Figure 1). We solve both problems here by showing that in both cases there is such a motion.

Physically, we think of a polygonal arc as a linkage or framework with hinges at its vertices, and rigid bars at its edges. The hinges can be folded as desired, but the bars must maintain their length and cannot cross. Motions of such linkages have been studied in

\footnotetext{
${ }^{*}$ Department of Mathematics, Cornell University, Ithaca, NY 14853, U.S.A., email: connelly@math. cornell.edu

${ }^{\dagger}$ MIT Laboratory for Computer Science, 200 Technology Square, Cambridge, MA 02139, USA, email: edemaine@mit .edu

${ }^{\ddagger}$ Institut für Informatik, Freie Universität Berlin, Takustraße 9, D-14195 Berlin, Germany, email: rote@ inf.fu-berlin.de
} 


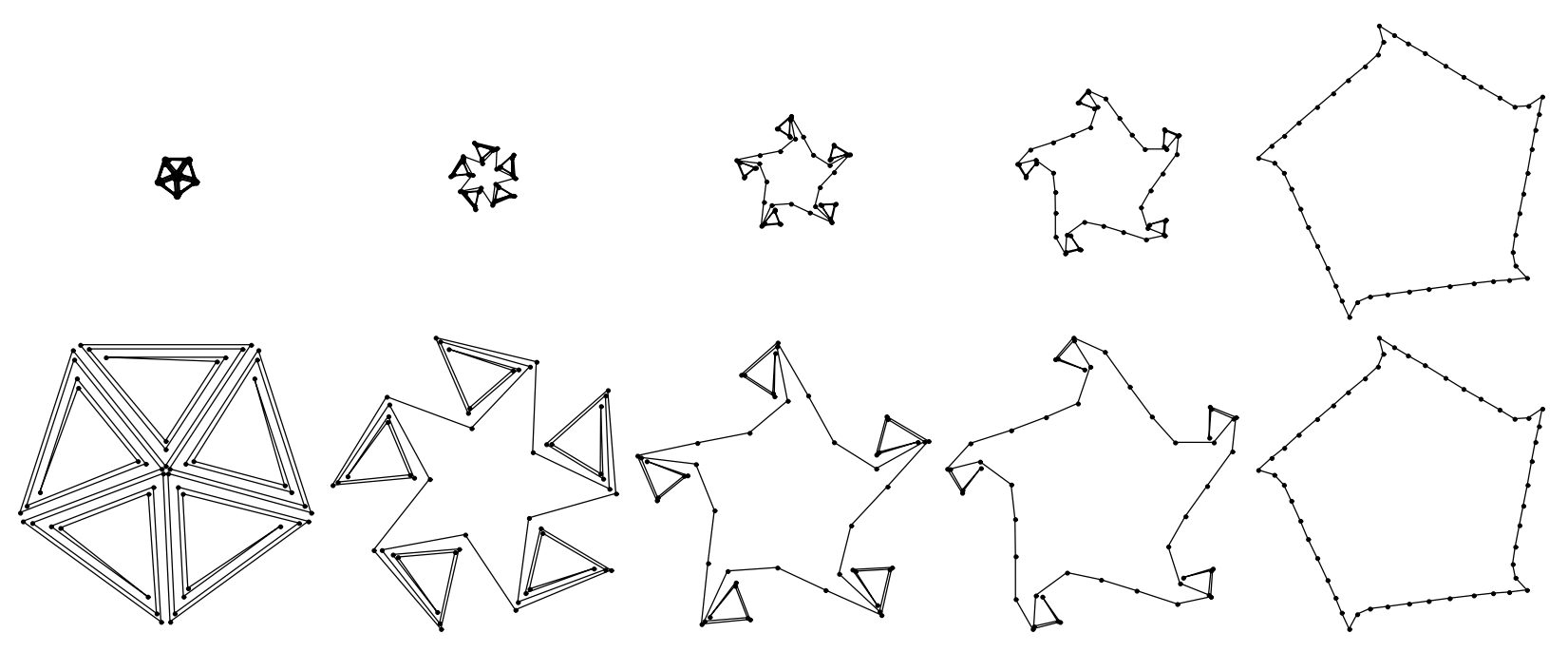

Figure 1: Two views of convexifying a polygon that comes from doubling each edge in a locked tree. The top snapshots are all scaled the same, and the bottom snapshots are scaled differently to improve visibility. More aminations can be seen at the world-wide-web pages of the first author. ${ }^{2}$

discrete and computational geometry $\left[\mathrm{BDD}^{+}\right.$01, Erd35, Grü95, LW95, Nag39, O'R98, Sal73, Tou99, Weg93, Weg96, Whi92b], in knot theory [CJ98, Mil94], and in molecular biology and polymer physics [FK97, MOS90, McM79, Mil94, SW88, SG72, Whi83]. Applications of this field include robotics, wire bending, hydraulic tube folding, and the study of macromolecule folding [O’R98, Tou99].

We say an arc is straightened by a motion if at the end of the motion it lies on a straight line. We say a polygonal simple closed curve (or cycle) is convexified by a motion if at the end of the motion it is a convex closed curve. All motions must be proper in the sense that no self-intersections are created, and each edge length is kept fixed. It is easy to see that if any cycle can be convexified by a motion, then any arc can be straightened by a motion: simply extend each arc to a cycle and convexify it. It is then easy to straighten the portion of the cycle that is the original arc.

It seems intuitively easy to straighten an entangled chain: just grab the ends and pull them apart. Similarly, a cycle might be opened by blowing air into it until it expands. But these methods have the difficulty that they might introduce singularities, where the arc or cycle intersects itself. Our approach is to use an expansive motion in which all distances between two vertices increase. We also show that the area of a polygon increases in such an expansive motion.

We consider the more general situation, which we call an arc-and-cycle set $A$, consisting of a finite number of polygonal arcs and polygonal cycles in the plane, with none of the arcs or cycles intersecting each other or having self-intersections. We say that $A$ is in an outer-convex configuration if each component of $A$ that is not contained in any cycle of $A$ is either straight (when it is an arc) or convex (when it is a cycle). The best we can hope for, in general, is to bring an arc-and-cycle set to an outer-convex configuration, because components nested

\footnotetext{
${ }^{2}$ Currently, http://db.uwaterloo.ca/ ${ }^{2}$ ddemain/linkage/
} 


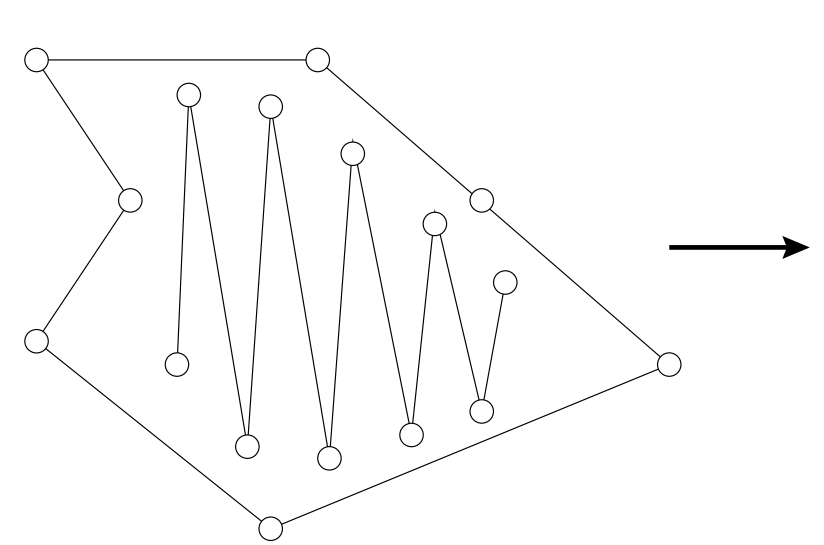

(a)

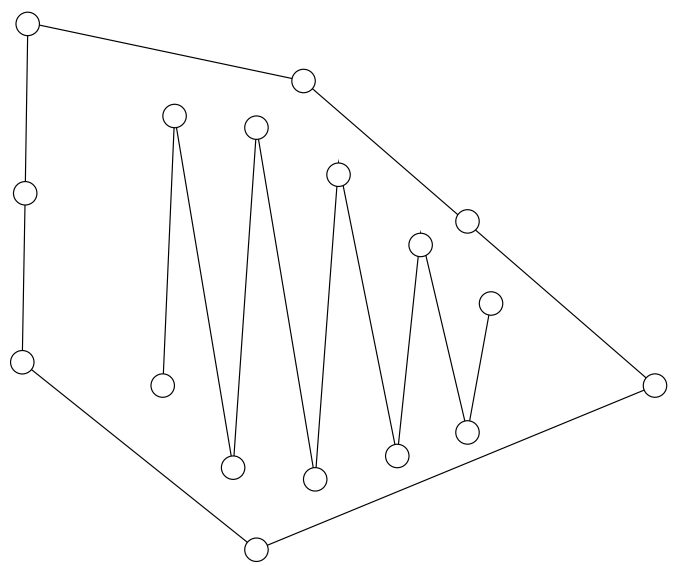

(b)

Figure 2: (a) The nested arc cannot be straightened because there is insufficient space in the containing cycle. (b) Once the containing cycle becomes convex, any expansive motion must move the arc and the cycle rigidly in unison.

within cycles cannot always be straightened or convexified; see Figure 2(a).

We say that a motion of an arc-and-cycle set $A$ is expansive if for every pair of vertices of $A$ the distance is monotonically nondecreasing over time, at all times either increasing or staying the same. In any expansive motion, once a cycle becomes convex, the cycle and any components it contains become a single rigid object; see Figure 2(b). (This fact is a consequence of Cauchy's Arm Lemma [Cau13, Cro97, SZ67].) Also, once two incident bars become collinear, they will remain so throughout any expansive motion, effectively acting as a single bar. We say that a motion is strictly expansive if the distance is constant between two vertices connected by a bar or straight chain of bars, and between two vertices on the boundary of or interior to a common convex cycle, but the distance between all other pairs of vertices monotonically strictly increases over time.

We say that the arc-and-cycle set $A$ has separated if there is a line $L$ in the plane such that $L$ is disjoint from $A$ and at least one component of $A$ lies on each side of $L$. Our main result is the following:

Theorem 1 Every arc-and-cycle set has a piecewise-differentiable proper motion to an outerconvex configuration. Moreover, the motion is strictly expansive until the arc-and-cycle set becomes separated.

We can also extend this result to insist that the motion be strictly expansive throughout the entire motion:

Theorem 2 Every arc-and-cycle set has a strictly expansive piecewise-differentiable proper motion to an outer-convex configuration.

For Theorem 2, the definition of the motion is actually even simpler than the one we use for Theorem 1, but unfortunately the proof is a great deal more complex. Thus we focus on the proof of Theorem 1. Theorem 2 has a similar proof outline, but it relies on a Boundedness 
Lemma (Lemma 10) whose proof is complicated. We include the proof of the Boundedness Lemma in Appendix A. Note that Theorems 1 and 2 only differ when there is more than one component in the arc-and-cycle set; the Boundedness Lemma is straightforward for a single arc or cycle.

In contrast to our results, in dimension three there are arcs that cannot be straightened and polygons that cannot be convexified $\left[\mathrm{BDD}^{+} 01, \mathrm{CJ} 98\right]$. In four and higher dimensions, no arcs, cycles, or trees can be locked, i.e., all arcs, cycles, or trees can be straightened or convexified, respectively. This is true because there are enough degrees of freedom and one can easily avoid any impending self-intersection by "moving around" it. An explicit unlocking algorithm for arcs and cycles in four dimensions was given in [CO99].

In the plane, there are examples of trees embedded in the plane that are locked in the sense that they cannot be properly moved so that the vertices lie nearly on a line [BDD $\left.{ }^{+} 98\right]$. In other words, there are two embeddings of the tree such that there is no proper motion from one configuration to the other. The important difference between trees and arc-and-cycle sets is that arc-and-cycle sets have maximum degree two. We have recently strengthened the example of $\left[\mathrm{BDD}^{+} 98\right]$ by constructing a locked tree with just one vertex of degree three and all other vertices of degree one or two [CDR02]. This shows that the restriction to arc-and-cycle sets in Theorem 1 is best possible.

\begin{tabular}{|l|l|l|}
\cline { 2 - 3 } \multicolumn{1}{c|}{} & \multicolumn{1}{c|}{ Arcs and Cycles } & \multicolumn{1}{c|}{ Trees } \\
\hline 2-D & Not lockable $[$ Theorem 1] & Lockable $\left[\right.$ BDD $\left.^{+} 98\right]$ \\
\hline 3-D & Lockable $\left[\right.$ BDD ${ }^{+} 01$, CJ98] & Lockable $\left[\right.$ BDD $^{+} 01$, CJ98] \\
\hline 4-D ${ }^{+}$ & Not lockable $[$CO99, CO01] & Not lockable $[$CO01] \\
\hline
\end{tabular}

Table 1: Summary of what types of linkages can be locked.

Whether every arc in the plane can be straightened, and whether every polygon in the plane can be convexified, have been outstanding open questions until now. The problems are natural, so they have arisen independently in a variety of fields, including topology, pattern recognition, and discrete geometry. We are probably not aware of all contexts in which the problem has appeared. To our knowledge, the first person to pose the problem of convexifying cycles was Stephen Schanuel. George Bergman learned of the problem from Schanuel during Bergman's visit to the State University of New York at Buffalo in the early 1970's, and suggested the simpler question of straightening arcs. As a consequence of this line of interest, the problems are included in Kirby's Problems in Low-Dimensional Topology [Kir97, Problem 5.18].

During the period 1986-1989, Ulf Grenander and the members of the Pattern Theory Group at Brown University explored various problems involving the probabilistic structure when generators (e.g., points and line segments) were transformed by diffeomorphisms (e.g., Euclidean transformations) subject to global constraints (e.g., avoiding intersections). For the purposes of Bayesian image understanding, they were interested in whether the process was ergodic, i.e., every configuration could be reached from any other. In particular, they proved this for polygonal cycles in which the roles of angles and lengths are reversed: angles are fixed but lengths may vary [GCK91, Appendix D, pp. 108-128]. Grenander posed the problems considered here in a seminar talk with the title "Can one understand shapes in 
nature?" at Indiana University, on March 27, 1987, and probably also on earlier occasions (according to personal communication with Allan Edmonds).

In the discrete and computational community, the problems were independently posed by William Lenhart and Sue Whitesides in March 1991 and by Joseph Mitchell in December 1992 (according to personal communications with Sue Whitesides and Joseph Mitchell). Sue Whitesides first posed this problem in a talk in 1991 [LW91]. In this community the problems were first published in a technical report in 1993 [LW93] and in a journal in 1995 [LW95].

Solutions were already known for the special cases of monotone cycles [BDL ${ }^{+} 99$ ] and star-shaped cycles [ELR ${ }^{+} 98$ ], and for certain types of "externally visible" arcs [BDST99].

A fairly large group of people, mentioned in the acknowledgments, was involved in trying to construct and prove or disprove locked arcs and cycles, at various times over the past few years. Typically, someone in the group would distribute an example that s/he constructed or was given by a colleague. We would try various motions that did not work, and we would often try proving that the example was locked because it appeared so! For some examples, it took several months before we found an unlocking motion. The main difficulty was that "simple" motions that change a few vertex angles at once, while easiest to visualize, seemed to be insufficient for unlocking complex examples. Amazingly, it also seemed that nevertheless there was always a global unlocking motion, and furthermore it was felt that there was a driving principle permitting "blowing up" of the linkage. This notion was formalized by the third author with the idea that perhaps an arc could be straightened via an expansive motion.

The tools that are applied here for the first time come from the theory of mechanisms and rigid frameworks. Arcs and cycles can be regarded as frameworks. See [AR78, AR79, Con80, Con82, Con93, CW96, CW93, CW82, CW94, GSS93, RW81, Whi84a, Whi84b, Whi87, Whi88, Whi92a] for relevant information about this theory.

Our approach is to prove that for any configuration there is an infinitesimal motion that increases all distances. Because of the nature of the arc-and-cycle set, this implies that there is a motion that works at least for a small expansive perturbation. We then combine these local motions into one complete motion. These notions are described in the rest of this paper. Section 3 proves the existence of infinitesimal motions using the nonexistence of certain stresses, a notion dual to infinitesimal motions for the underlying framework. The analysis of these stresses uses a lifting theorem from the theory of rigidity that was known to James Clerk Maxwell and Luigi Cremona [CW82, CW93, Whi82] in the nineteenth century. Section 4 shows how to maneuver through the space of local motions to find a global motion with the desired properties.

A short version of this paper was presented at the 41st Annual Symposium on Foundations of Computer Science in November 2000 [CDR00].

$5: 38$

$5: 39$

$5: 40$

$5: 41$

$5: 42$

\section{Basics}

A linkage or bar framework $G(\mathbf{p})$ is a finite graph $G=(V, E)$ without loops or multiple edges, together with a corresponding configuration $\mathbf{p}=\left(\mathbf{p}_{1}, \ldots, \mathbf{p}_{n}\right)$ of $n$ distinct points in the plane, where $\mathbf{p}_{i}$ corresponds to vertex $i \in V$. (For convenience we assume $V=\{1, \ldots, n\}$.) The edges of $G$ constitute the set $E$ and correspond to the bars in the framework, i.e., the links 
of a linkage. Arc-and-cycle sets are a particular kind of bar framework in which the graph $G$ is a disjoint union of paths and cycles.

A flex or motion of $G(\mathbf{p})$ is a set of continuous functions $\mathbf{p}(t)=\left(\mathbf{p}_{1}(t), \ldots, \mathbf{p}_{n}(t)\right)$, defined for $0 \leq t \leq 1$, such that $\mathbf{p}(0)=\mathbf{p}$ and the Euclidean distance $\left\|\mathbf{p}_{i}(t)-\mathbf{p}_{j}(t)\right\|$ is constant for each $\{i, j\} \in E$. We are interested in finding a motion of the arc-and-cycle set with the additional property that it is strictly expansive.

\subsection{Expansiveness}

We begin with some basic properties of expansive motions. Namely, we will show that if a motion expands the distance between all pairs of vertices, it also expands the distance between all pairs of points on the arc-and-cycle framework. One consequence of this property is a key reason why we use expansive motions: they automatically avoid self-intersection. To prove the property, we need the following known basic geometric tool, which will also be useful later:

Lemma $\mathbf{1}$ In the plane, suppose that a point $\mathbf{c}$ is contained in the closed triangle $\mathbf{p}_{1} \mathbf{p}_{2} \mathbf{p}_{3}$, and $\mathbf{p}_{1}$ and $\mathbf{p}_{2}$ are farther from another point $\mathbf{q}_{3}$ than from $\mathbf{p}_{3}$, i.e.,

$$
\left\|\mathbf{q}_{3}-\mathbf{p}_{2}\right\| \geq\left\|\mathbf{p}_{3}-\mathbf{p}_{2}\right\| \text { and }\left\|\mathbf{q}_{3}-\mathbf{p}_{1}\right\| \geq\left\|\mathbf{p}_{3}-\mathbf{p}_{1}\right\|
$$

Then $\mathbf{c}$ is also farther from $\mathbf{q}_{3}$ than from $\mathbf{p}_{3}$, i.e., $\left\|\mathbf{q}_{3}-\mathbf{c}\right\| \geq\left\|\mathbf{p}_{3}-\mathbf{c}\right\|$, with equality only if both inequalities of (1) are equalities.

Proof: Refer to Figure 3. The circular disk $C_{0}$ centered at $\mathbf{c}$ with radius $\left\|\mathbf{p}_{3}-\mathbf{c}\right\|$ is contained in the union of the circular disks $C_{1}$ with center $\mathbf{p}_{1}$ and radius $\left\|\mathbf{p}_{3}-\mathbf{p}_{1}\right\|$, and $C_{2}$ with center $\mathbf{p}_{2}$ and radius $\left\|\mathbf{p}_{3}-\mathbf{p}_{2}\right\|$. This implies the result, because $\mathbf{q}_{3}$ must be outside $C_{1}$ and $C_{2}$. See also [Con82] for a proof in terms of tensegrities.

Corollary 1 Any expansive motion of an arc-and-cycle set only increases the distance between two points on the arc-and-cycle set (each either a vertex or on a bar). In particular, there can be no self-intersections.

Proof: Refer to Figure 4. First, the result is obvious if the two points are both vertices of the arc-and-cycle set, by definition of expansiveness. Second, consider the distance between a vertex $\mathbf{p}_{3}$ of the arc-and-cycle set and a point $\mathbf{c}$ on a bar $\mathbf{p}_{1} \mathbf{p}_{2}$ of the arc-and-cycle set. Expansiveness implies that $\mathbf{p}_{3}$ only gets farther from $\mathbf{p}_{1}$ and $\mathbf{p}_{2}$, so by Lemma $1, \mathbf{p}_{3}$ only gets farther from $\mathbf{c}$. Third, consider the distance between $\mathbf{c}$ (again on the bar $\mathbf{p}_{1} \mathbf{p}_{2}$ ) and another point $\mathbf{d}$ on a bar $\mathbf{p}_{4} \mathbf{p}_{5}$ of the arc-and-cycle set. Substituting $\mathbf{p}_{4}$ and $\mathbf{p}_{5}$ as options for $\mathbf{p}_{3}$ in the previous argument, we know that $\mathbf{p}_{4}$ and $\mathbf{p}_{5}$ only get farther from $\mathbf{c}$. Applying Lemma 1 with $\mathbf{c}$ playing the role of $\mathbf{p}_{3}$, we obtain that $\mathbf{c}$ can only get farther from $\mathbf{d}$. 


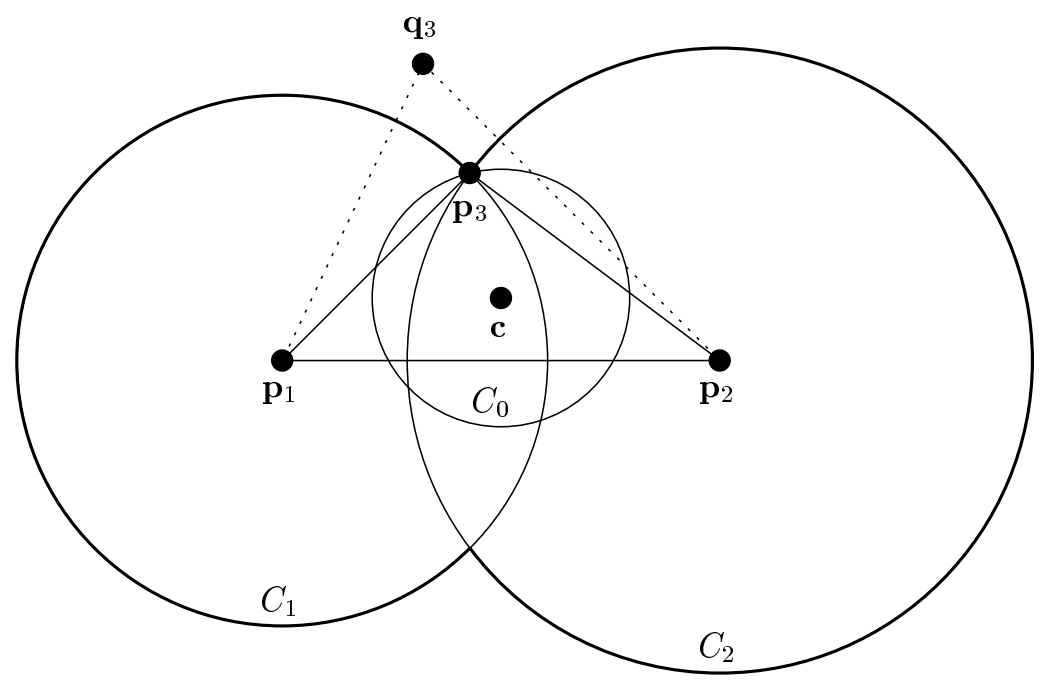

Figure 3: Illustration of Lemma 1.
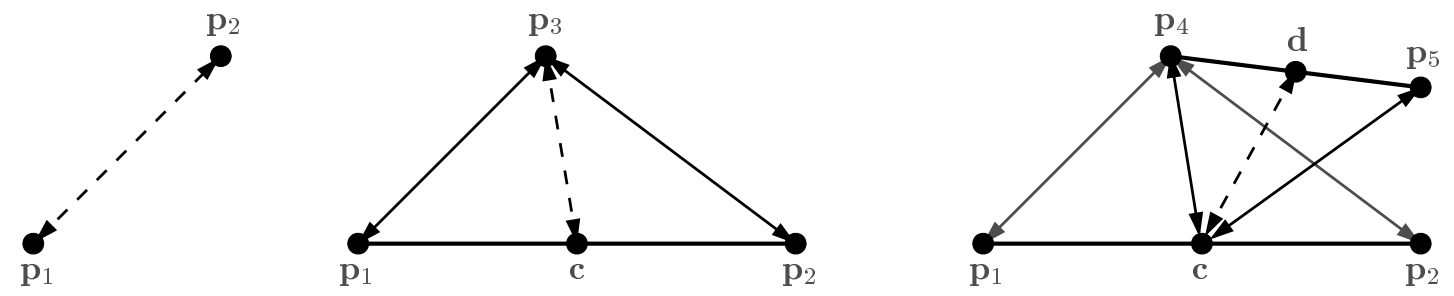

Figure 4: Illustration of the three cases of Corollary 1: (left) two vertices $\mathbf{p}_{1}, \mathbf{p}_{2}$; (middle) one vertex $\mathbf{p}_{3}$ and one point $\mathbf{c}$ on a bar $\mathbf{p}_{1} \mathbf{p}_{2}$; (right) one point $\mathbf{c}$ on a bar $\mathbf{p}_{1} \mathbf{p}_{2}$ and another point $\mathbf{d}$ on a bar $\mathbf{p}_{4} \mathbf{p}_{5}$. Bold edges denote bars, and arrows denote expansion; dashed arrows are derived from solid arrows.

\subsection{The Framework $G_{A}(\mathbf{p})$}

Given an arc-and-cycle set $A$ that we would like to move to an outer-convex configuration, we make four modifications to $A$. The first three modifications simplify the problem by removing a few special cases that are easy to deal with; see Figure 5. The fourth modification will bring the problem of finding a strictly expansive motion into the area of tensegrity theory. In the end we will have defined a new framework, $G_{A}(\mathbf{p})$, which we will use throughout the rest of the proof.

Modification 1: Remove straight vertices. First we show that our arc-and-cycle set can be assumed to have no straight vertices, i.e., vertices with angle $\pi$. Furthermore, if during an expansive motion of the arc-and-cycle set we find that a vertex becomes straight, we can proceed by induction. For once the arc-and-cycle set has a straight subarc of more than one bar, we can coalesce this subarc into a single bar, thereby preserving the straightness of the subarc throughout the motion once it becomes straight. This reduces the number of bars and the number of vertices in the framework. By induction, this reduced framework has a motion according to Theorem 1, and such a motion extends directly to the original 


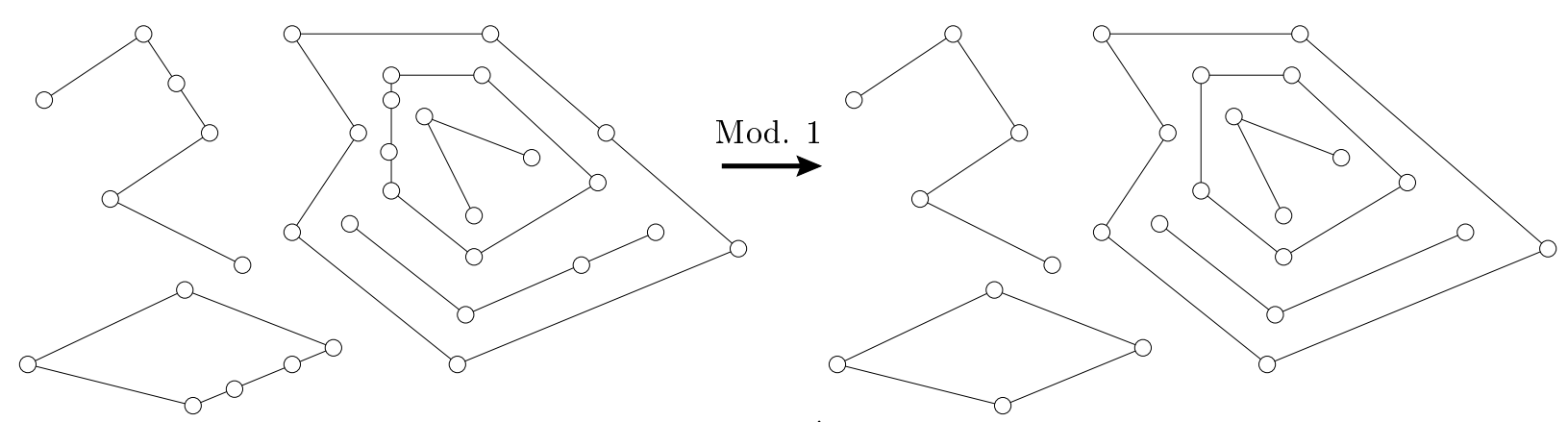

(a)

Mod. 2

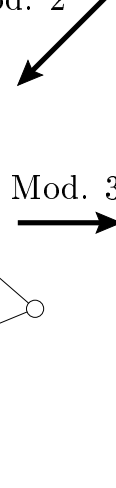

(c) (b)

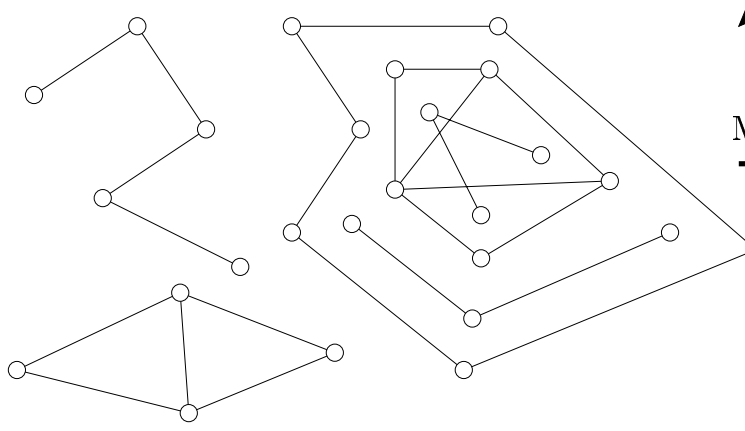

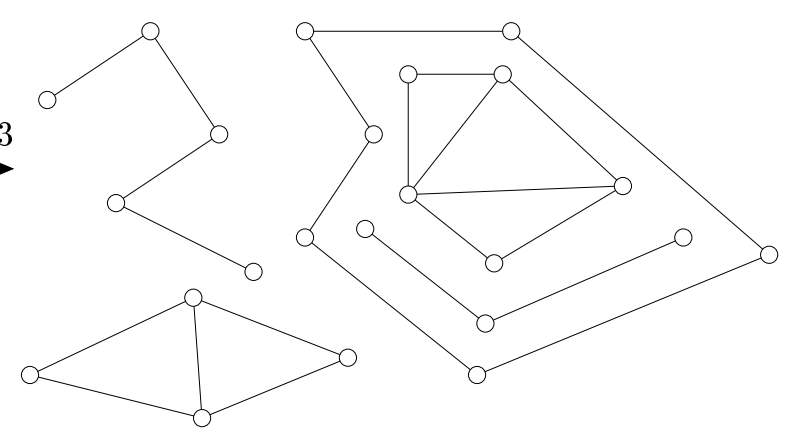

(d)

Figure 5: (a) Original arc-and-cycle framework. (b) With straight vertices removed. (c) With convex cycles rigidified. (d) With components nested within convex cycles removed.

framework. The resulting motion is also strictly expansive by Corollary 1 .

Modification 2: Rigidify convex polygons. Once a cycle becomes convex, we no longer have to expand it, indeed it is impossible to expand, so we hold it rigid from that point on. Of course, we allow a convex cycle to translate or rotate in the plane, but its vertex angles are not allowed to change. This can be directly modeled in the bar framework by introducing bars in addition to the arc-and-set cycle. Specifically, we add the edges of a triangulation of a cycle once that cycle becomes convex. We deal with the contents of the cycle in the next modification.

Modification 3: Remove components nested within convex cycles. The previous modification did not address the fact that components can be nested within cycles. Once a cycle becomes convex, not only can we rigidify it, but we can also rigidify any nested components, and treat them as moving in synchrony with the convex cycle. We do this by removing from the framework any components nested within a convex cycle. Assuming there were some nested components to deal with, this results in a framework with fewer vertices and fewer bars. By induction, this reduced framework has a motion according to Theorem 1. This motion can be extended to apply to the original framework by defining nested components to follow the rigid motion of the containing convex cycle (rigid by Modification 2). By the following consequence of Lemma 1 , the resulting motion is also strictly expansive. 
Lemma 2 Extending a motion to apply to components nested within convex cycles preserves strict expansiveness.

Proof: Consider some vertex $\mathbf{c}$ on a component inside some convex cycle, and a vertex $\mathbf{p}_{3}$ outside the cycle. We first consider the case that $\mathbf{p}_{3}$ does not lie inside another convex cycle. Extend the ray from $\mathbf{p}_{3} \mathbf{c}$ beyond $\mathbf{c}$, and let $\mathbf{p}_{1} \mathbf{p}_{2}$ be the edge through which this ray exits the cycle. Thus, $\mathbf{c}$ is in the triangle $\mathbf{p}_{1}, \mathbf{p}_{2}, \mathbf{p}_{3}$, so Lemma 1 applies, and the distance $\mathbf{p}_{3} \mathbf{c}$ increases.

For two points $\mathbf{c}_{1}$ and $\mathbf{c}_{2}$ in two different cycles $C_{1}$ and $C_{2}$, we extend the ray $\mathbf{c}_{1} \mathbf{c}_{2}$ to identify the edge $\mathbf{p}_{1} \mathbf{p}_{2}$ on $C_{2}$ where the ray leaves $C_{2}$. From the first part of the proof we conclude that $\mathbf{c}_{1} \mathbf{p}_{1}$ and $\mathbf{c}_{1} \mathbf{p}_{2}$ increase, and by Lemma 1 , the distance $\mathbf{c}_{1} \mathbf{c}_{2}$ increases.

Modification 4: Add struts. In order to model the expansive property we need, we apply the theory of tensegrity frameworks, in which frameworks can consist of both bars and "struts." In contrast to a bar, which must stay the same length throughout a motion, a strut is permitted to increase in length, or stay the same length, but cannot shorten. The last modification adds a strut between nearly every pair of vertices in the framework. The exceptions are those vertices already connected by a bar, and vertices on a common convex cycle, because in both cases we cannot hope to strictly increase the distance.

Final framework: $G_{A}(\mathbf{p})$. The above modifications define a tensegrity (bar-and-strut) framework $G_{A}(\mathbf{p})$ in terms of the arc-and-cycle set $A$. Specifically, assume that $A$ has no straight vertices (Modification 1) and no components nested within convex cycles (Modification 3). We call such an arc-and-cycle set reduced. We define the set of bars, $B$, to consist of the bars from the arc-and-cycle set together with the bars forming the rigidifying triangulation of each convex cycle (Modification 2). The set $S$ of struts consists of all vertex pairs that are not connected by a bar in $B$ and which do not belong to a common convex cycle (Modification 4). See Figure 6 for an example of $A$ and the resulting bar-and-strut framework $G_{A}(\mathbf{p})$. (The rightmost framework $G_{A}^{\prime}\left(\mathbf{p}^{\prime}\right)$ will be defined later.)
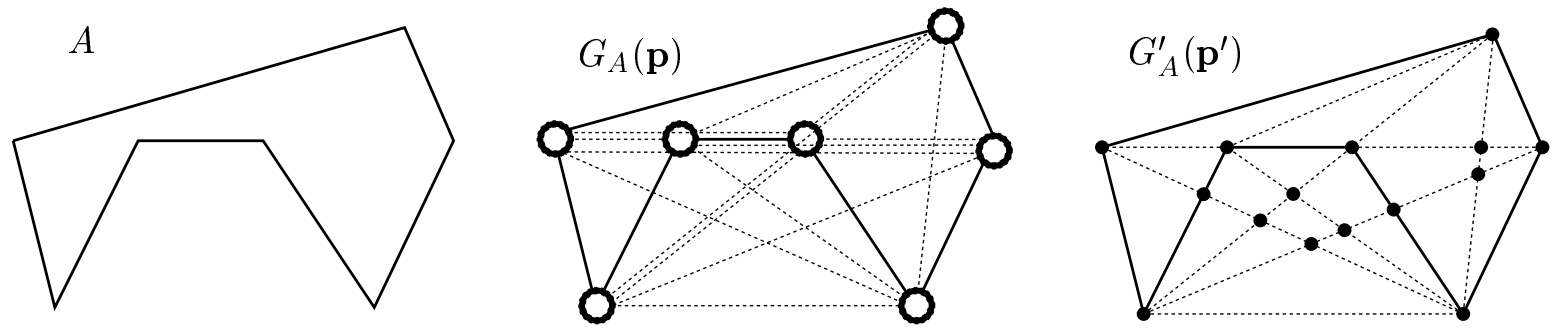

Figure 6: Construction of the frameworks $G_{A}(\mathbf{p})$ and $G_{A}^{\prime}\left(\mathbf{p}^{\prime}\right)$. Solid lines denote bars, and dashed lines denote struts.

Our goal in the proof of Theorem 1 is to find a motion such that all bars maintain their length, while all struts strictly increase in length, in other words, a motion of $G_{A}(\mathbf{p})$ that is strict on all struts. 
Thus, we want to find a motion $\mathbf{p}(t)$ for $0 \leq t \leq 1$ such that $\mathbf{p}(0)=\mathbf{p}$ and

$$
\begin{array}{ll}
\frac{d}{d t}\left\|\mathbf{p}_{j}(t)-\mathbf{p}_{i}(t)\right\|=0 & \text { for }\{i, j\} \in B, \\
\frac{d}{d t}\left\|\mathbf{p}_{j}(t)-\mathbf{p}_{i}(t)\right\|>0 & \text { for }\{i, j\} \in S .
\end{array}
$$

10:03

10:04

10:05

10:06

10:07

10:08

Differentiating the squared distances $\left\|\mathbf{p}_{j}(t)-\mathbf{p}_{i}(t)\right\|^{2}=\left(\mathbf{p}_{j}(t)-\mathbf{p}_{i}(t)\right) \cdot\left(\mathbf{p}_{j}(t)-\mathbf{p}_{i}(t)\right)$ and denoting the velocity vectors by $\mathbf{v}_{i}(t):=\frac{d}{d t} \mathbf{p}_{i}(t)$, we obtain the following equivalent conditions.

$$
\begin{array}{ll}
\left(\mathbf{v}_{j}(t)-\mathbf{v}_{i}(t)\right) \cdot\left(\mathbf{p}_{j}(t)-\mathbf{p}_{i}(t)\right)=0 & \text { for }\{i, j\} \in B, \\
\left(\mathbf{v}_{j}(t)-\mathbf{v}_{i}(t)\right) \cdot\left(\mathbf{p}_{j}(t)-\mathbf{p}_{i}(t)\right)>0 & \text { for }\{i, j\} \in S .
\end{array}
$$

Intuitively, the first-order change in the distance between vertex $i$ and $j$ is modeled by projecting the velocity vectors onto the line segment between the two vertices; see Figure 7.

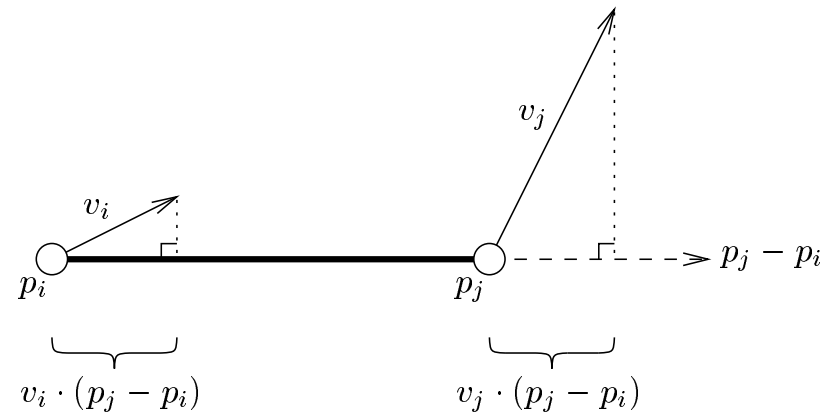

Figure 7: The dot product $\left(\mathbf{v}_{j}(t)-\mathbf{v}_{i}(t)\right) \cdot\left(\mathbf{p}_{j}(t)-\mathbf{p}_{i}(t)\right)$ is zero if the distance between $\mathbf{p}_{i}$ and $\mathbf{p}_{j}$ stays the same to the first order, positive if the distance increases to the first order, and negative if the distance decreases to the first order.

\subsection{Infinitesimal Motions}

A strict infinitesimal motion or strict infinitesimal flex $\mathbf{v}=\left(\mathbf{v}_{1}, \ldots, \mathbf{v}_{n}\right)$ specifies the first derivative of a strictly expansive motion at time 0 . In other words, it assigns a velocity vector $\mathbf{v}_{i}$ to each vertex $i$ so that it preserves the length of the bars to the first order, and strictly increases the length of struts to the first order. More precisely, a strict infinitesimal motion must satisfy

$$
\begin{aligned}
& \left(\mathbf{v}_{j}-\mathbf{v}_{i}\right) \cdot\left(\mathbf{p}_{j}-\mathbf{p}_{i}\right)=0 \text { for }\{i, j\} \in B, \\
& \left(\mathbf{v}_{j}-\mathbf{v}_{i}\right) \cdot\left(\mathbf{p}_{j}-\mathbf{p}_{i}\right)>0 \text { for }\{i, j\} \in S,
\end{aligned}
$$

where $\mathbf{p}_{i}$ denotes the initial position of vertex $i$.

In the next section, we prove that such a strict infinitesimal motion always exists. In Section 4 we show how this leads to motions for small amounts of time. These motions are then shown to continue globally until the configuration reaches an outer-convex configuration.

\section{Local Motion}

Recall that an arc-and-cycle set is called reduced if adjacent collinear bars have been coalesced, and components nested within convex cycles have been removed. In this section, we 
prove the following:

Theorem 3 For any reduced arc-and-cycle set $A$ there is an infinitesimal motion $\mathbf{v}$ of the corresponding bar-and-strut framework $G_{A}(\mathbf{p})$ satisfying $(2)$.

The proof will go through a sequence of transformations from motions to stresses, and from there to polyhedral terrains, to which geometric reasoning is finally applied. A second, independent, but no less indirect proof of Theorem 3 follows from the results about the structure of the expansion cone in [RSS02, Theorem 4.3].

\subsection{Equilibrium Stresses}

The equations and inequalities in (2) form a linear feasibility problem that is common for tensegrity frameworks. In order to solve this problem, it is helpful to apply linearprogramming duality and consider the equivalent dual problem. We discuss the duality first in terms of equilibrium stresses in tensegrity frameworks, and later reconnect it to linearprogramming duality.

A stress in a framework $G(\mathbf{p})$ is an assignment of a scalar $\omega_{i j}=\omega_{j i}$ to each edge $\{i, j\}$ of $G$ (a bar or strut). A negative scalar means that the edge is pushing on its two endpoints by an equal amount, a positive value means that the edge is pulling on its endpoints by an equal amount, and zero means that the edge induces no force. The whole stress is denoted by $\omega=\left(\ldots, \omega_{i j}, \ldots\right)$. We say that the stress $\omega$ is an equilibrium stress if each vertex $i$ of $G$ is in equilibrium, i.e., stationary subject to the the forces from the incident edges:

$$
\sum_{j:\{i, j\} \in B \cup S} \omega_{i j}\left(\mathbf{p}_{j}-\mathbf{p}_{i}\right)=0
$$

We say that the stress $\omega$ is proper if furthermore, for all struts $\{i, j\}, \omega_{i j} \leq 0$. That is, struts can carry only zero or negative stress. There is no sign condition for bars: they can carry zero, positive, or negative stress. Thus, only bars can carry positive stress. (Terminology and sign conditions have not always been uniform in the literature. An equilibrium stress is also called a self-stress or simply a stress. All stresses that we deal with are equilibrium stresses.)

A trivial example of an equilibrium stress is the zero stress in which every edge is assigned a scalar of zero. All other stresses are called nonzero. To prove Theorem 3, we use the following duality principle connecting nonzero equilibrium stresses and infinitesimal motions:

Lemma 3 If the only proper equilibrium stress in a bar-and-strut framework is the zero stress, then the framework has an infinitesimal motion satisfying (2).

This equivalence is a standard technique in the theory of rigidity. See [CW96, Theorem 2.3.2] for a similar statement. For completeness, we give a brief proof here based on linear programming duality:

Proof: To make it easier to take the dual of the linear feasibility problem defined by (2), we write a linear program in standard form. First we add an otherwise pointless objective 
function of $0=0 \cdot \mathbf{v}$. Then we rescale the velocities $\mathbf{v}$ in (2) to obtain the following equivalent linear program:

$$
\begin{array}{ll}
\operatorname{minimize} & 0 \cdot \mathbf{v} \\
\text { subject to } & \left(\mathbf{v}_{j}-\mathbf{v}_{i}\right) \cdot\left(\mathbf{p}_{j}-\mathbf{p}_{i}\right)=0 \text { for }\{i, j\} \in B, \\
& \left(\mathbf{v}_{j}-\mathbf{v}_{i}\right) \cdot\left(\mathbf{p}_{j}-\mathbf{p}_{i}\right) \geq 1 \text { for }\{i, j\} \in S,
\end{array}
$$

We wish to show that the framework has an infinitesimal motion, which is equivalent to this linear program having a feasible solution, that is, an optimal solution of value 0 . By linearprogramming duality (the Farkas lemma), it suffices to show that the dual linear program

$$
\begin{array}{lll}
\text { maximize } & \sum_{\{i, j\} \in S} \bar{\omega}_{i j} & \\
\text { subject to } & \sum_{j:\{i, j\} \in B \cup S} \bar{\omega}_{i j}\left(\mathbf{p}_{j}-\mathbf{p}_{i}\right)=0 & \text { for } i \in V, \\
& \bar{\omega}_{i j}=\bar{\omega}_{j i} & \text { for }\{i, j\} \in B \cup S, \\
& \bar{\omega}_{i j} \geq 0 & \text { for }\{i, j\} \in S,
\end{array}
$$

has an optimal solution of value 0 . If we express the constraints of this linear program in terms of negated dual variables $\omega_{i j}=-\bar{\omega}_{i j}$, they specify precisely a proper equilibrium stress. Thus, it suffices to show that every proper equilibrium stress has $\omega_{i j}=0$ for all $\{i, j\} \in S$. In particular, it suffices to show that every proper equilibrium stress is identically zero.

The important consequence of this lemma is that, in order to prove the desired Theorem 3 , it suffices to prove the following:

Theorem 4 The framework $G_{A}(\mathbf{p})$ corresponding to a reduced arc-and-cycle set $A$ has only the zero proper equilibrium stress.

\subsection{Planarization}

To prove that only the zero equilibrium stress exists (i.e., to prove Theorem 4), we use another tool in rigidity called the Maxwell-Cremona theorem. Before we can apply this tool, we need to transform the framework $G_{A}(\mathbf{p})$ into a planar framework $G_{A}^{\prime}\left(\mathbf{p}^{\prime}\right)$. (Refer to the framework on the right of Figure 6.) We introduce new vertices at all intersection points between edges of $G_{A}(\mathbf{p})$. We subdivide every bar and strut at every vertex through which it goes, be it an existing vertex of $G_{A}(\mathbf{p})$ or a newly added intersection vertex. Overlapping collinear edges will result in multiple edges; such multiple are merged into one edge. We define the resulting framework $G_{A}^{\prime}\left(\mathbf{p}^{\prime}\right)$ to have bars precisely covering the bars of $G_{A}(\mathbf{p})$. All the other edges of $G_{A}^{\prime}\left(\mathbf{p}^{\prime}\right)$ are struts. $G_{A}^{\prime}\left(\mathbf{p}^{\prime}\right)$ is planar in the sense that two edges (bars or struts) intersect only at a common endpoint.

A natural concern is that the added vertices in this modification introduce additional freedom in finding infinitesimal motions, so they may not transfer directly to infinitesimal motions in the original framework. Nonetheless, the planar framework $G_{A}^{\prime}\left(\mathbf{p}^{\prime}\right)$ is effectively equivalent to the original framework $G_{A}(\mathbf{p})$ in the sense of equilibrium stresses. Indeed, the 
following stronger statement holds. We call a stress outer-zero if the only edges that carry a nonzero stress are edges of convex cycles and edges interior to convex cycles. Otherwise, an edge exterior to all convex cycles carries a nonzero stress, and we call the stress outer-nonzero.

Lemma 4 If $G_{A}(\mathbf{p})$ has a nonzero proper equilibrium stress $\omega$, then $G_{A}^{\prime}\left(\mathbf{p}^{\prime}\right)$ has an outernonzero proper equilibrium stress $\omega^{\prime}$.

Proof: During the modifications to $G_{A}(\mathbf{p})$ that made $G_{A}^{\prime}\left(\mathbf{p}^{\prime}\right)$, we modify $\omega$ to make $\omega^{\prime}$ as follows. When we subdivide an edge $\{i, j\}$ with stress $\omega_{i j}$, each edge $\{k, l\}$ of the subdivision gets the stress $\omega_{i j}\left\|p_{i}-p_{j}\right\| /\left\|p_{k}-p_{l}\right\|$. (The ratio of lengths is necessary because $\omega_{i j}$ is a weight, and the actual force comes from scaling by the length of the edge $\{i, j\}$; see (3).) When merging several edges, we add the corresponding stresses. The resulting stress is in equilibrium because edges meet in opposing pairs at the added vertices, and because summation preserves force. The stress is also proper because a strut in $G_{A}^{\prime}\left(\mathbf{p}^{\prime}\right)$ is made up only of struts from $G_{A}(\mathbf{p})$, and the sum of nonnegative numbers is nonnegative. It only remains to check that positive and negative stresses do not completely cancel during the merging process, and that the stress is furthermore outer-nonzero.

First we prove that some strut $\{i, j\}$ of $G_{A}(\mathbf{p})$ carries a negative stress. In other words, $G_{A}(\mathbf{p})$ cannot be stressed only on its bars; in particular, a framework consisting exclusively of arcs, cycles, and triangulated convex cycles cannot carry a nonzero stress. This follows because, in any such bar framework, there is a vertex $\mathbf{v}$ with degree at most two; in particular, every triangulated convex cycle has a degree-two vertex (an ear). Because the framework is reduced, the two bars incident to $\mathbf{v}$ are not parallel, so these two bars cannot carry stress while satisfying equilibrium at $\mathbf{v}$. Removing them and proceeding inductively with the rest of the framework, we conclude that the stress is zero on the whole bar framework. Hence, the bars alone cannot carry a nonzero stress, so some strut $\{i, j\} \in G_{A}(\mathbf{p})$ must have a nonzero stress.

The conditions of Theorem 3 enforce that no angles at vertices of the arc-and-cycle set are $\pi$ or 0 : an angle of $\pi$ would create a straight subarc of two bars (contradicting the assumption that the framework is reduced), and an angle of 0 would violate simplicity. Thus, no strut of $G_{A}(\mathbf{p})$ is completely covered by bars. Therefore, for the strut $\{i, j\}$ of $G_{A}(\mathbf{p})$ that carries a negative stress, some portion of it in $G_{A}^{\prime}\left(\mathbf{p}^{\prime}\right)$ will also have a negative stress, because a negative stress can only be canceled by a stress on a bar. In particular, $\omega^{\prime}$ must be nonzero.

Furthermore, if the strut $\{i, j\}$ is exterior to all convex cycles in $A$, we have that $\omega^{\prime}$ is outer-nonzero. Now suppose that $\{i, j\}$ is partially interior to convex cycles in $A$ (by construction, the strut cannot be entirely within convex cycles of $A$ ). Then there is a portion of $\{i, j\}$ with the property that it is incident to a convex cycle and exterior to all convex cycles in $A$. This portion must be uncovered by bars, because no bar in $A$ has this property, and the only additional bars in $G_{A}(\mathbf{p})$ are interior to convex cycles. Hence, the corresponding strut in $G_{A}^{\prime}(\mathbf{p})$ carries a negative stress, so $\omega^{\prime}$ is outer-nonzero in all cases.

Thus, to prove that the original framework $G_{A}(\mathbf{p})$ has only the zero proper equilibrium stress, it suffices to prove that the planar framework $G_{A}^{\prime}\left(\mathbf{p}^{\prime}\right)$ has only outer-zero proper equilibrium stresses. 


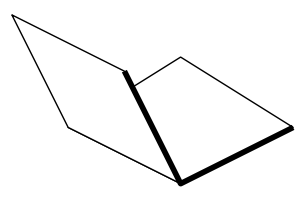

(a) Valley

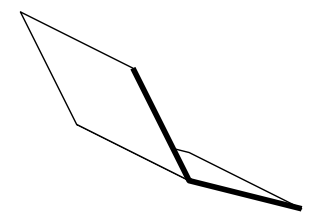

(b) Valley

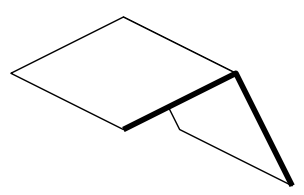

(c) Mountain

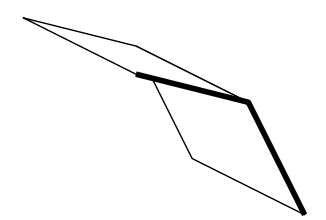

(d) Mountain

Figure 8: Valleys and mountains in a polyhedral terrain. The thick edges indicate the intersection with a vertical plane.

\subsection{Maxwell-Cremona Theorem}

To prove that only outer-zero equilibrium stresses exist, we employ the Maxwell-Cremona correspondence between equilibrium stresses in planar frameworks and three-dimensional polyhedral graphs that project onto these frameworks. When the vertices and edges of a planar framework are removed from the plane the resulting connected regions are called the faces of the framework. A polyhedral graph or polyhedral terrain $\Gamma$ comes from lifting a planar framework into three dimensions - that is, assigning a $z$ coordinate (positive or negative) to each vertex in the framework - such that the vertices of each face of the framework (including the exterior face) remain coplanar. Thus, each face of the framework lifts to a planar polygon in 3-space. The polyhedral surface $\Gamma$ is then the graph of a piecewise-linear continuous function of two variables that is linear on the faces determined by $G_{A}^{\prime}\left(\mathbf{p}^{\prime}\right)$.

Consider an edge $\{i, j\}$ in a planar framework, separating faces $F$ and $F^{\prime}$. We distinguish whether this edge lifts in $\Gamma$ to a "valley," "mountain," or "flat" edge according to its dihedral angles; see Figure 8. More formally, let $z(\mathbf{p})=\mathbf{a} \cdot \mathbf{p}+b$ for $\mathbf{p} i n F$ and $z(\mathbf{p})=\mathbf{a}^{\prime} \cdot \mathbf{p}+b^{\prime}$ for $\mathbf{p} i n F$ be the two linear functions specifying the graph $\Gamma$ on $F$ and $F^{\prime}$. Thus, a and $\mathbf{a}^{\prime}$ are vectors in (the dual space of) $\mathbb{R}^{2}$, and $b$ and $b^{\prime}$ are real numbers. A straightforward calculation reveals that the vector $\mathbf{a}-\mathbf{a}^{\prime}$ in $\mathbb{R}^{2}$ must be perpendicular to the edge $\{i, j\}$ :

$$
\mathbf{a}-\mathbf{a}^{\prime}=\omega_{i j} \mathbf{e}_{i j}^{\perp}
$$

where $\mathbf{e}_{i j}^{\perp}$ is a vector in $\mathbb{R}^{2}$ of the same length as the vector $\mathbf{p}_{j}-\mathbf{p}_{i}$, perpendicular to it, and pointing from $F$ towards $F^{\prime}$. We call the edge $\{i, j\}$ a valley if $\omega_{i j}<0$, a mountain if $\omega_{i j}>0$, and flat if $\omega_{i j}=0$.

These definitions are illustrated in Figure 8. In particular, if two sides of an edge both "go up" in $z$, as in Figure 8(a), then the edge is a valley; however, the converse does not hold (so a valley might not carry water), as shown in Figure 8(b). Similarly, if the two sides of an edge both "go down" in $z$ (Figure 8(c)), then the edge must be a mountain, but not all mountains have this property (Figure $8(\mathrm{~d})$ ).

Theorem 5 (Maxwell-Cremona Theorem) (i) For every polyhedral graph $\Gamma$ that projects to a planar bar framework $G(\mathbf{p})$, the stress $\omega$ defined by (6) forms an equilibrium stress on $G(\mathbf{p})$.

(ii) For every proper equilibrium stress $\omega$ in a planar framework $G(\mathbf{p}), G(\mathbf{p})$ can be lifted to a polyhedral graph $\Gamma$ such that (6) holds for all edges. In particular, edges with positive stress lift to valleys, edges with negative stress lift to mountains, and edges with no stress lift to flat edges. Furthermore, $\Gamma$ is unique up to addition of affine-linear functions. 


\subsection{Main Argument}

The zero equilibrium stress corresponds to a trivial polyhedral graph in which all faces are coplanar (i.e., defined by a single linear function). More generally, an outer-zero equilibrium stress corresponds to an outer-flat polyhedral graph that is flat on every edge exterior to all convex cycles. Therefore, to prove that all equilibrium stresses of the planar framework are outer-zero, and hence prove Theorem 4, it suffices to show that all polyhedral graphs projecting to the planar framework are outer-flat.

More precisely, consider any polyhedral graph $\Gamma$ that projects to the planar framework $G_{A}^{\prime}\left(\mathbf{p}^{\prime}\right)$ with the property that all struts are lifted to valleys or flat edges (because struts can carry only negative or zero stress), and bars are lifted to valleys, mountains, or flat edges. We need to show that nonflat edges can only appear within or on the boundary of convex cycles. Because we may add an arbitrary affine-linear function, we may conveniently assume that the exterior face of $\Gamma$ is on the $x y$-plane. Thus the problem is to show that $\Gamma$ does not lift off the $x y$-plane any vertex of $G_{A}^{\prime}\left(\mathbf{p}^{\prime}\right)$ except possibly vertices interior to convex cycles of $A$.

One simple fact that we will need is the following:

Lemma 5 Any mountain in the polyhedral graph $\Gamma$ projects to a bar in the planar framework $G_{A}^{\prime}\left(\mathbf{p}^{\prime}\right)$.

Proof: A strut can only carry negative or zero stress, so by Theorem 5 it can only lift to a valley or a flat edge.

We now come to the heart of our proof, the proof of Theorem 6. It is here that we finally show that the stress must be outer-zero, by looking at the maximum of any Maxwell-Cremona lift. The following statement immediately implies Theorem 4 and hence Theorem 3:

Theorem 6 Let $M$ denote the region in the $x y$-plane where the $z$ value attains its maximum in the polyhedral graph $\Gamma$. Then $M$ contains every face of the planar framework $G_{A}^{\prime}\left(\mathbf{p}^{\prime}\right)$ that is exterior to all convex cycles.

$M$ is a nonempty union of faces, edges, and vertices of the planar framework $G_{A}^{\prime}\left(\mathbf{p}^{\prime}\right)$. Consider the boundary $\partial M$, which may be empty if $M$ fills the whole plane. Because points in $M$ lift to maximum height, all edges of $\partial M$ must lift to mountains. Thus by Lemma 5 , all edges of $\partial M$ must be bars in the framework. Hence, $\partial M$ consists of disjoint vertices, paths of edges, and complete cycles of the arc-and-cycle set, together with a subset of the triangulations of the convex components. Figure 9 shows typical cases of all possibilities. We will show that the only case in Figure 9 that can actually occur is $(\ell)$, in which $\partial M$ includes a convex cycle and $M$ includes the local exterior of that cycle (and possibly some of its interior).

Our main technique for arriving at a contradiction in all cases except $(\ell)$ is that of slicing the polyhedral graph. Consider a plane $\Pi$ that is parallel to the $x y$-plane and just below the maximum $z$ coordinate of $\Gamma$. (By "just below" we mean that $\Pi$ is above all vertices of $\Gamma$ 


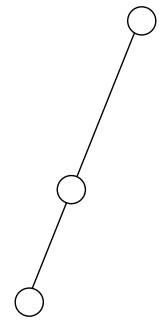

(b)

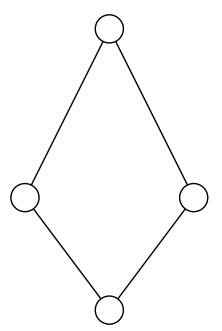

(g)

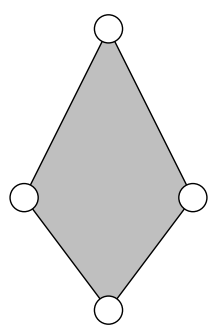

(h)

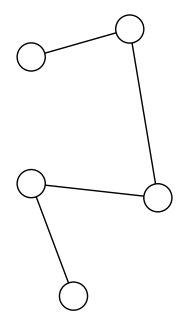

(c)

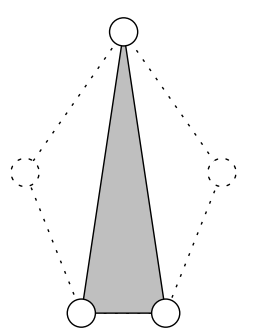

(i)

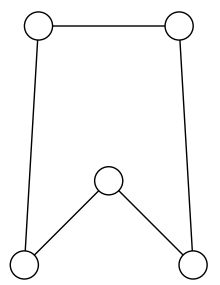

(d)

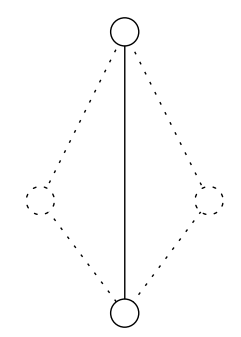

(j)

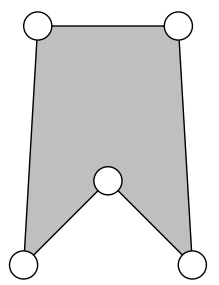

(e)

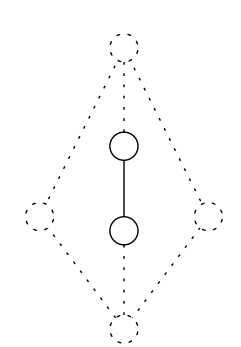

$(\mathrm{k})$

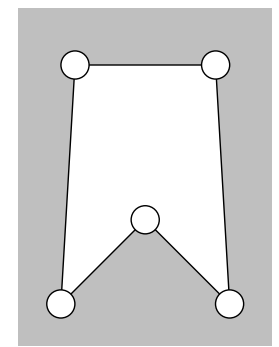

(f)

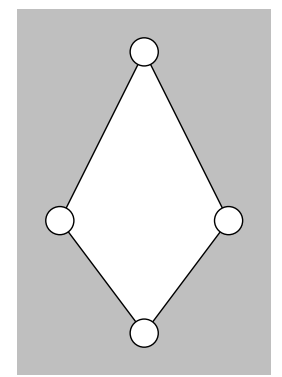

$(\ell)$

Figure 9: Hypothetical connected components of $\partial M$ and their relation to $M$. Solid lines are edges of $\partial M$; white regions are absent from $M$; and shaded regions are present in $M$. (a) An isolated vertex. (b) A straight subarc. (c) A nonstraight subarc. (d) A nonconvex cycle. (e) A nonconvex cycle and its local interior. (f) A nonconvex cycle and its local exterior. $(\mathrm{g}-\mathrm{k})$ Various situations with a convex cycle. $(\ell)$ The only possible case: A convex cycle, its local exterior, and possibly some of its interior.

not at the maximum $z$ coordinate.) Now take the intersection of $\Pi$ with the surface $\Gamma$, and project this intersection to the $x y$-plane. The resulting set $X$ is shown in Figure 10 for the various cases.

The set $X$ captures several properties of the polyhedral graph $\Gamma$. First note that because $X$ is the boundary of a small neighborhood of $M$ in the plane, it is a disjoint union of cycles. It is also polygonal. Each edge of $X$ corresponds to a face of $\Gamma$, and each vertex of $X$ corresponds to an edge of $\Gamma$. The angle at a vertex of $X$ (on the side interior to $M$ ) determines the type of edge corresponding to that vertex: the angle is $\pi$ (straight) if the edge is flat, less than $\pi$ (convex) if the edge is a mountain, and more than $\pi$ (reflex) if the edge is a valley.

The basic idea is to show that $X$ has "many" convex angles, and apply Lemma 5 to prove that the framework has "too many" bars. The key fact underlying the proof is that the original arc-and-cycle set has maximum bar-degree two: every vertex is incident to at most two bars. In the planar framework $G_{A}^{\prime}\left(\mathbf{p}^{\prime}\right)$, only vertices $\mathbf{v}$ of convex cycles can have bar-degrees greater than two, and these bars are contained in a convex wedge from $\mathbf{v}$.

Our proof deals with all cases at once. To illustrate the essence of the proof, we first describe it for a subcase of case (a) in which one component of $\partial M$ is a single vertex $\mathbf{v}$ that does not belong to a convex cycle. In this case, one component of $X$ is a planar polygonal cycle $P$ that is star-shaped around $\mathbf{v}$, that is, every point on the boundary of $P$ is visible 


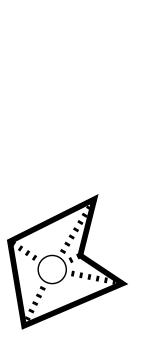

(a)

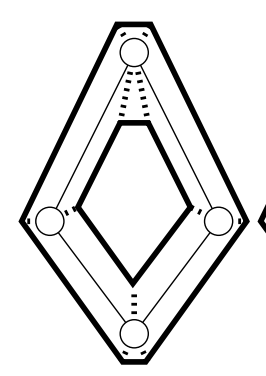

(g)

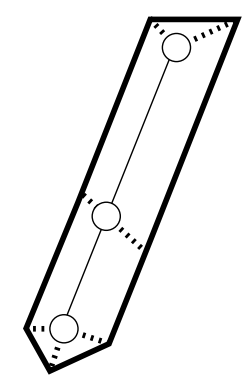

(b)

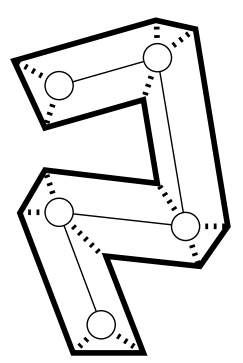

(c)

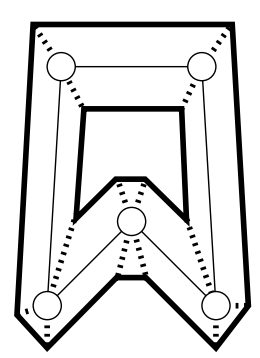

(d)

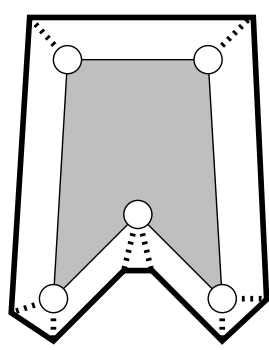

(e)

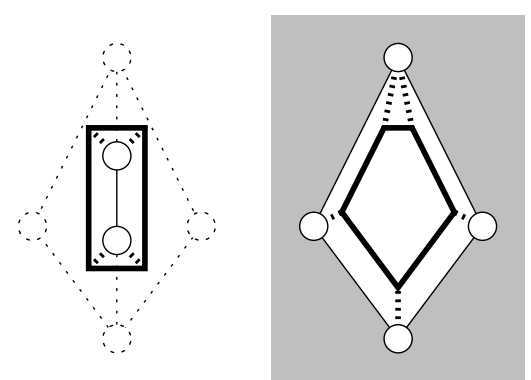

$(\mathrm{k})$

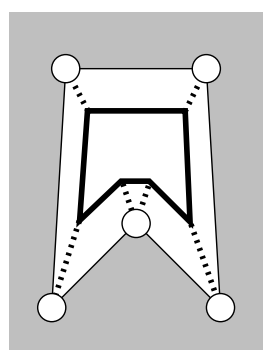

(f)
(1)

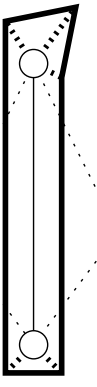

(j)

Figure 10: Slicing the polyhedral graph $\Gamma$ just below the maximum $z$ coordinate, in each case corresponding to those in Figure 9. Thick lines denote the slice intersection $X$, and thick dotted lines denote the corresponding edges in the polyhedral graph $\Gamma$.

from v. In particular, $P$ is a planar polygon with positive area and no self-intersections. Every such polygon has at least three convex vertices. (To see this, define the turn angle at a vertex to be $\pi$ minus the interior angle, so it is positive for convex angles and negative for reflex angles, and always strictly between $-\pi$ and $\pi$. Because the turn angles of a planar polygon sum to $2 \pi$, and the maximum turn angle of a vertex is $<\pi$, every polygon has at least three vertices with positive turn angles.) These three convex vertices correspond to three mountains in $\Gamma$, all incident to a common vertex $v$. By Lemma 5 , there are three bars incident to $v$, contradicting the maximum-degree-two property for vertices not on convex cycles. Therefore, this subcase of case (a) cannot exist.

The general reason that cases $(\mathrm{a}-\mathrm{k})$ cannot exist is the following:

Lemma 6 Let $\mathbf{v}$ be a vertex on the boundary of $M$, and let $b_{1}, \ldots, b_{k}$ be the bars incident to $\mathbf{v}$ in cyclic order. Consider a small disk D around $\mathbf{v}$.

(1) If there is an angle of at least $\pi$ at $\mathbf{v}$ between two consecutive bars, say $b_{i}$ and $b_{i+1}$, then the pie wedge $P$ of $D$ bounded by $b_{i}$ and $b_{i+1}$ belongs to $M$ (see Figure 11).

(2) If there are no bars or only one bar incident to $\mathbf{v}$, i.e., $k \leq 1$, then the entire disk $D$ belongs to $M$. (This can be viewed as a special case of (1).)

Proof: (1) Because there are no bars in the pie wedge $P$, and hence no edges of $\partial M$ in $P$, $P$ must be completely contained in or disjoint from $M$. Assume to the contrary that $P$ is disjoint from $M$. Then the intersection of the slice $X$ with the pie wedge $P$ is a star-shaped 

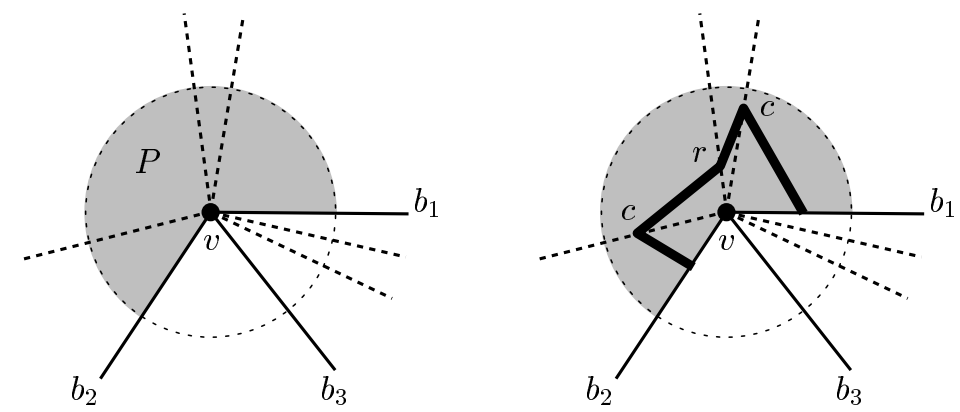

Figure 11: (Left) Illustration of Lemma 6: solid lines are bars, dotted lines are struts, and the shaded pie wedge $P$ must be contained in $M$. (Right) Illustration of the proof; the thick lines form the portion of $X$ inside $P$, and the symbols $c$ and $r$ denote convex and reflex vertices, respectively.

polygonal arc around $\mathbf{v}$ starting from a point on $b_{i}$ and ending at a point on $b_{i+1}$. By the properties of $X$, convex vertices on this arc correspond to mountains emanating from $\mathbf{v}$, and reflex vertices correspond to valleys emanating from $\mathbf{v}$. Because the angle of the pie wedge $P$ is at least $\pi$, the arc must have at least one convex vertex in $P$. (The turn angles along the arc must sum to a positive number, so some vertex must have a positive turn angle.) By Lemma 5 , there must be a bar in $P$, a contradiction.

(2) If $k=1$, the bars $b_{i}$ and $b_{i+1}$ coincide, and the same proof applies. The star-shaped polygonal arc becomes a star-shaped polygonal cycle, which must have at least two convex vertices not on $b_{i}=b_{i+1}$. If $k=0, X$ also has a star-shaped polygonal cycle around $\mathbf{v}$, which must have at least three convex vertices, yet $\mathbf{v}$ has no incident bars.

Note that this lemma applies to every vertex in our planar framework $G_{A}^{\prime}\left(\mathbf{p}^{\prime}\right)$, because every vertex either has bar-degree at most two or is a vertex of a convex cycle, and in either case there is a nonconvex angle between two consecutive bars.

One can immediately verify that the examples in Figure 10(a-k) contradict Lemma 6. For example, applying the lemma to any vertex of $\partial M$ shows that $M$ should contain a positive two-dimensional area incident to that vertex. This immediately rules out cases $(\mathrm{a}-\mathrm{d}),(\mathrm{g})$, $(\mathrm{j})$, and $(\mathrm{k})$.

A general proof is also easy with Lemma 6 in hand:

Proof (Theorem 6): Consider first a degree- 0 or degree- 1 vertex $\mathbf{v}$ in $\partial M$. (Such a point would appear when $M$ has a component that is an isolated point or an arc of bars.) Because Lemma 6 applies to every vertex of the framework, we know that some positive two-dimensional area in the vicinity of $\mathbf{v}$ belongs to $M$, contradicting that $\mathbf{v}$ has degree 0 or 1 in $\partial M$. This rules out cases $(\mathrm{a}-\mathrm{c})$ and $(\mathrm{j}-\mathrm{k})$.

It follows that $\partial M$ is a union of cycles. A component of $\partial M$ can be of two kinds:

(1) If it is formed from the edges of a convex cycle and its triangulation, Lemma 6 applies to any vertex in it, and we conclude that $M$ contains the face of the framework immediately exterior to the cycle. This rules out cases $(\mathrm{g}-\mathrm{i})$.

(2) If it consists of a complete nonconvex cycle, we can apply Lemma 6 to some convex vertex and to some reflex vertex (they must both exist), and we conclude that $M$ 
contains both the face of the framework immediately interior and the face immediately exterior to the cycle. This rules out cases $(d-f)$.

In the end, the only faces of the framework that can be missing from $M$ are those interior to convex cycles (case $(\ell)$ ). This completes the proof of Theorem 6 and of Theorems 4 and 3 .

\section{Global Motion}

In this section, we combine the infinitesimal motions into a global motion, thereby proving Theorem 1, the main theorem. In overview, Theorem 3 establishes the existence of some direction of motion $\mathbf{v}$. We select a unique vector $\mathbf{v}=f(\mathbf{p})$ for each configuration $\mathbf{p}$ as the solution of a convex optimization problem (7-9). We then set up the differential equation

$$
\frac{d}{d t} \mathbf{p}(t)=f(\mathbf{p}(t))
$$

The solution of this differential equation moves the linkage to a configuration where an angle between two bars becomes straight. At this point we merge the two bars and continue with the reduced framework that has one vertex less. This procedure is iterated until the framework is outer-convex and no further expansive motion is possible.

It is convenient for the proof of Theorem 1 to effectively pin an edge in the configuration. Choose any edge, say $\left\{\mathbf{p}_{1}, \mathbf{p}_{2}\right\}$, that is a bar. During the motion we will arrange matters so that this bar is stationary.

We now go into the details of the proof. We use the following nonlinear minimization problem to define a unique direction $\mathbf{v}$ for every configuration $\mathbf{p}$ of a reduced arc-and-cycle set.

$$
\begin{aligned}
\operatorname{minimize} & \sum_{i \in V}\left\|\mathbf{v}_{i}\right\|^{2}+\sum_{\{i, j\} \in S} \frac{1}{\left(\mathbf{v}_{i}-\mathbf{v}_{j}\right) \cdot\left(\mathbf{p}_{i}-\mathbf{p}_{j}\right)-\left\|\mathbf{p}_{j}-\mathbf{p}_{i}\right\|} \\
\text { Subject to } \quad\left(\mathbf{v}_{j}-\mathbf{v}_{i}\right) \cdot\left(\mathbf{p}_{j}-\mathbf{p}_{i}\right) & >\left\|\mathbf{p}_{j}-\mathbf{p}_{i}\right\|, \quad \text { for }\{i, j\} \in S \\
\left(\mathbf{v}_{j}-\mathbf{v}_{i}\right) \cdot\left(\mathbf{p}_{j}-\mathbf{p}_{i}\right) & =0, \text { for }\{i, j\} \in B \\
\mathbf{v}_{1}=\mathbf{v}_{2} & =0
\end{aligned}
$$

The restrictions (8) place a uniform constraint on the growth of the struts $S$ : the derivative of the length of each strut must be larger than 1. Since the system (2) is homogeneous, the system (8-9) is feasible for any choice of right-hand sides in (8). This particular right-hand side has been chosen for convenience in the proof.

The objective function (7) includes the norm of $\mathbf{v}$ as a quadratic term, plus a barrier-type penalty term that keeps the solution away from the boundary (8) of the feasible region. This penalty term is necessary to achieve a smooth dependence of the solution on the data. The objective function is strictly convex because it is a sum of strictly convex functions, of the form $x^{2}$ for a variable $x$, and convex functions, of the form $1 / f\left(x_{1}, x_{2}, x_{3}, x_{4}\right)$ where $f$ is an affine function in four variables that is guaranteed to be positive. Because the objective 
function is strictly convex, and it goes to infinity if $\mathbf{v}$ increases to infinity or approaches the boundary of condition (8), there is a unique solution $\mathbf{v}$ for every $\mathbf{p}$; we denote this solution by $f(\mathbf{p})$.

The function $f(\mathbf{p})$ is defined on an open set $U \subset \mathbb{R}^{2 n}$ that is characterized by the conditions of Theorem 3: no angles are $0^{\circ}$ or $180^{\circ}$, no vertex touches a bar, and at least one cycle is nonconvex or at least one open arc is not straight.

\subsection{Smoothness}

We will show that $f$ is differentiable in the domain $U$. This follows from the stability theory of convex programming under equality constraints, as applied to parametric optimization problems of the type

$$
\min \left\{g(p, x): x \in \Omega(p) \subseteq \mathbb{R}^{n}, A(p) x=b(p)\right\}
$$

where $A(p)$ is an $m \times n$ matrix and $b(p)$ is an $m$-vector. The objective function $g$, the domain $\Omega(p)$, and the linear constraints $(A, b)$ depend on a parameter $p$ that ranges over an open region $U \subseteq \mathbb{R}^{k}$.

For such an optimization problem, the following lemma establishes the smooth dependence of the solution vector on the problem-definition data $A(p)$ and $b(p)$.

Lemma 7 Suppose that the following conditions are satisfied in the optimization problem (11).

(a) The objective function $g(p, x)$ is twice continuously differentiable and strictly convex as a function of $x \in \Omega(p)$, with a positive definite Hessian $H_{g}$, for every $p \in U$.

(b) The domain $\Omega(p)$ is an open set, for every $p \in U$.

(c) The rows of the constraint matrix $A(p)$ are linearly independent, for every $p \in U$.

(d) The problem-definition data $A(p)$ and $b(p)$ and the gradient $\nabla g$ of $g$ with respect to $x$ are continuously differentiable in $p$, for $p \in U$.

(e) The optimum point $x^{*}(p)$ of the problem (11) exists for every $p \in U$ (and is unique, by $(\mathrm{a}))$.

Then $x^{*}(p)$ is continuously differentiable in $U$.

Proof: The proof is based on the implicit function theorem and follows the standard lines of the proofs in this area; cf. [BS74, in particular Section 4] or [Fia76, Theorem 2.1] for more general theorems where inequalities are also allowed. For the benefit of the reader, we sketch the proof here. From (a) and (e) it follows that $x^{*}$ can be found as part of the unique solution $\left(x^{*}, \lambda\right)$ of the system of equations $h(p, x, \lambda)=0$ that represents the first-order necessary optimality conditions. Specifically, $\lambda$ is a $k$-element vector of Lagrange multipliers, and $h: U \times \mathbb{R}^{n+k} \rightarrow \mathbb{R}^{n+k}$ is given by

$$
h=\left(\begin{array}{c}
\nabla g-\lambda^{\mathrm{T}} A^{\mathrm{T}} \\
A x-b
\end{array}\right) .
$$


The implicit function theorem guarantees the local existence of $x(p)$ (and $\lambda(p)$ ) as a solution of $h(p, x(p), \lambda(p))=0$ in a neighborhood of $x$ if the Jacobian $J=\partial h / \partial(x, \lambda)$ is an invertible matrix for every $p \in U$. Moreover, differentiability of $x(p)$ is ensured if $h$ is continuously differentiable. The Jacobi matrix is given by

$$
J=\frac{\partial h(p, x, \lambda)}{\partial(x, \lambda)}=\left(\begin{array}{cc}
H_{g} & A^{\mathrm{T}} \\
A & 0
\end{array}\right) .
$$

Differentiability of $h$ follows from assumption (d); we only have to check that $J$ is invertible. By assumption (a), $H_{g}$ is positive definite and hence invertible. Thus

$$
\operatorname{det} J=\operatorname{det} H_{g} \cdot \operatorname{det}\left(-A H_{g}^{-1} A^{\mathrm{T}}\right) .
$$

By assumption (c), $A$ has full row rank, and because $H_{g}$ is positive definite, so is $A H_{g}^{-1} A^{\mathrm{T}}$. Therefore $\operatorname{det} J \neq 0$.

Lemma $8 f$ is differentiable on $U$.

Proof: The objective function is the sum of the quadratic function $\sum\left\|\mathbf{v}_{i}\right\|^{2}$, which has a positive definite (constant) Hessian, and additional smooth convex terms, and therefore assumption (a) of Lemma 7 holds, as well as the part of assumption (d) regarding $g$. The feasible domain $\Omega$ is defined by the inequalities (8), and because the inequalities are strict, $\Omega$ is an open set, so assumption (b) holds. The problem-definition data $A$ and $b$ are defined by the linear constraints (9) and (10). Both are clearly continuously differentiable, verifying the remaining half of assumption (d). Assumption (e) follows from the existence of an infinitesimal motion (Theorem 3).

It only remains to check assumption (c), the linear independence of the defining equations. Note that (10) implies (9) for the edge $\{1,2\}$, so the latter equation is redundant and can be omitted without changing the problem. In order to show that the remaining equations of the system (9-10) are linearly independent, we check that they have a solution for any choice of right-hand sides. We prove this by induction on the number of vertices. We select a vertex $i \notin\{1,2\}$ that is incident to at most two bars $\{i, j\},\{i, k\}$. The existence of such a vertex follows from an extension of an argument in the proof of Lemma 4: a framework consisting exclusively of arcs, cycles, and triangulated convex cycles, contains at least two non-adjacent vertices with degree at most two (unless the framework consists of a single triangle; in that case there are three vertices of degree two).

If the vertex $i$ is incident to two bars, they cannot be parallel. Thus, the corresponding unknown vector $\mathbf{v}_{i}$ appears in at most two equations in which the scalar products with two vectors $\mathbf{p}_{i}-\mathbf{p}_{j}$ and $\mathbf{p}_{i}-\mathbf{p}_{k}$ are taken; because these vectors are not parallel, there is always a solution for $\mathbf{v}_{i}$ regardless of the values of the other variables. If follows that any solution of the system without the variable $\mathbf{v}_{i}$ can be extended to $\mathbf{v}_{i}$. The reduced system is of the same form as the original system, and therefore we can apply induction. 


\subsection{Solving the Differential Equation and Proving Theorem 1}

Differentiability of $f$ on $U$ is sufficient to ensure that the initial-value problem

$$
\frac{d}{d t} \mathbf{p}(t)=f(\mathbf{p}(t)), \quad \mathbf{p}(0)=\mathbf{p}_{0}
$$

has a (unique) maximal solution $\mathbf{p}(t), 0 \leq t<T$, that cannot be extended beyond some positive bound $T \leq \infty$; see for example [Wal96, Section II.XXI]. This means that one of three cases occurs:

(a) $\mathbf{p}(t)$ exists for all $t$, i.e., $T=\infty$.

(b) $T$ is finite, and $\mathbf{p}(t)$ becomes unbounded as $t \rightarrow T$.

(c) $T$ is finite, and $\mathbf{p}(t)$ approaches the boundary of $U$ as $t \rightarrow T$.

The last case (c) is the case we want: at the boundary of $U$, some angle becomes straight, and we can reduce the linkage. The other two cases must be avoided: In case (a), the motion of the framework slows down and never reaches the limit of an outer-convex configuration. Case (b) can arise only with multiple disjoint components: the components could repel and fly away from each other faster than they straighten or convexify, thus never reaching an outer-convex configuration.

Case (a) can be excluded very easily. By assumption, the bar-and-strut framework $G_{A}(\mathbf{p})$ has some strut $\{i, j\}$ between two points in the same component of the bar framework; their distance increases at least with rate 1 , by (8), but it is bounded from above because $i$ and $j$ are linked by a sequence of bars. It follows that the solution cannot exist indefinitely and $T$ must be finite.

To deal with case (b), we apply the following observation.

Lemma 9 If an arc-and-cycle set $A$ is not separated, then its diameter is bounded by the total length of all edges of $A$.

This can be proved easily by induction on the number of components of $A$. Since some vertices are pinned at the origin, case (b) implies that $A$ must become separated by a line $L$. At this point, we stop the motion defined by (12) and treat the two parts into which $L$ separates the components of $A$ independently and recursively. Unfortunately, the guarantee for the expansive property between different members of the partition is lost.

We are left with case (c). We show that $\mathbf{p}(t)$ converges as $t \rightarrow T$. Observe that all pairwise distances of vertices $\mathbf{p}(t)$ are monotonically increasing, and by condition (10) $\mathbf{p}_{1}$ and $\mathbf{p}_{2}$ are fixed during the motion. Thus, all other vertices are determined up to reflection (through $\mathbf{p}_{1} \mathbf{p}_{2}$ ), so the whole configuration is determined up to reflection. Thus $\mathbf{p}(t) \rightarrow \mathbf{p}$ for some configuration $\mathbf{p}$ as $t \rightarrow T$. The configuration $\mathbf{p}$ is on the boundary of $U$ and thus must have some vertex with a straight angle. Then we inductively continue with a simpler linkage. This completes the proof of Theorem 1.

In this proof, the easy exclusion of possibility (b), that the motion becomes unbounded, depends crucially on the fact that the diameter of $A$ is bounded, and the motion is stopped as soon as there is a separating line. Boundedness is valid even without this precaution, as stated in the following lemma. 
Lemma 10 (Boundedness Lemma) Let $\mathbf{p}(t)$ be the motion given by the differential equation (12), where $\mathbf{v}=f(\mathbf{p})$ is given as the solution of the optimization problem (7-9). Then the motion of every vertex $i$ is bounded:

$$
\left\|\mathbf{p}_{i}(t)-\mathbf{p}_{i}(0)\right\| \leq \int_{0}^{t}\left\|\mathbf{v}_{i}(t)\right\| d t \leq K_{B, S, \mathbf{p}_{0}}(t),
$$

where $K_{B, S, \mathbf{p}_{0}}(t)$ is an explicit function of that depends only on the combinatorial structure of the arc-and-cycle set $(B$ and $S)$ and on the initial configuration $\mathbf{p}_{0}$.

Note that the definition of $\mathbf{v}$ does not involve the pinning constraints (10). The lemma implies that it is not necessary to treat separated components separately. The proof of the lemma is complicated, and it is given in Appendix A.

\subsection{Alternative Approaches}

There are many ways to select a local motion $\mathbf{v}$ among the feasible local motions whose existence is guaranteed by Theorem 3. We have chosen one possibility in Equations (7-10) that is most convenient for the proof.

As a possible alternative approach, we might consider a linear programming problem, with some arbitrary artificial linear objective function $\mathbf{c}$, and some linear normalization condition to ensure boundedness, pinning down some bar $\left(i_{1}, i_{2}\right) \in B$ :

$$
\begin{aligned}
\operatorname{minimize} & \sum_{i \in V} \mathbf{c}_{i} \cdot \mathbf{v}_{i} \\
\text { subject to } & \left(\mathbf{v}_{j}-\mathbf{v}_{i}\right) \cdot\left(\mathbf{p}_{j}-\mathbf{p}_{i}\right)=0 \text { for }\{i, j\} \in B, \\
& \left(\mathbf{v}_{j}-\mathbf{v}_{i}\right) \cdot\left(\mathbf{p}_{j}-\mathbf{p}_{i}\right) \geq 0 \text { for }\{i, j\} \in S, \\
& \sum_{i \in V} \mathbf{d}_{i} \cdot \mathbf{v}_{i}=1 \\
& \mathbf{v}_{i_{1}}=\mathbf{v}_{i_{2}}=0
\end{aligned}
$$

We have given up strict expansiveness in (14), The set of vectors given by (13), (14), and (16) forms a polyhedral cone $C$. Theorem 3 guarantees that there are nonzero solutions. One can check that the pinning constraints (16) ensure that the cone is pointed. The idea is now to use an extreme ray of the cone $C$ for the motion. A vector $\mathbf{d}$ can be found which ensures that the feasible set (13-16) is a bounded set. Any basic feasible solution of the linear program will correspond to an extreme ray of the cone $C$. It will have a few inequalities of (14) fulfilled with equality. The resulting framework obtained by inserting "artificial" bars corresponding to the nonbasic inequalities of (14), will have a unique vector of velocities $\mathbf{v}$ subject to the normalization constraint (15). This means that the framework is a mechanism, allowing one degree of freedom; as the mechanism follows this forced motion, all nonfixed distances will increase, at least for some time.

So one follows the paradigm of parametric linear programming: The optimal basic feasible solution will continue to remain feasible as the coefficients $\mathbf{p}_{i}$ in the constraints (14) change smoothly. At some point, one of these constraints will threaten to become violated: this is the time to make a pivot, exchanging one of the artificial bars for a new one which allows the motion to be continued. 
The above discussion has ignored several issues, such as possible degeneracy of the linear program. However, this approach might be more attractive from a conceptual, as well as practical, point of view.

Streinu [Str00] has found a class of such mechanisms, so-called pseudo-triangulations. These structures have several nice properties; for example, they form a planar framework of bars. Streinu [Str00] has proved that a polygonal arc can be opened by a sequence of at most $O\left(n^{2}\right)$ motions, where each motion is given by the mechanism of a single pseudotriangulation.

The polyhedral cone $C$ mentioned above has been more thoroughly investigated in Rote, Santos, and Streinu [RSS02]. In particular, they studied the so-called expansion cone, which is simply defined by the equations (14) for all pairs of points $i$ and $j$. The extreme rays of this cone are closely related to the set of all pseudo-triangulations of a point set.

\subsection{Comparison of Approaches}

The approach based on mechanisms might avoid some of the numerical difficulties associated with solving the optimization problem (7-9). For example, consider a spiral $n$-bar arc winding around a unit square in layers of thickness $\varepsilon$ (Figure 12). Basically, a strut deep inside the spiral cannot increase in length quickly before an outer strut increases significantly. But in the solution of (8-9), the inner strut lengths must increase at unit speed; a rough estimate shows that this causes the outermost vertex to move with an exponential speed of at least $(1 / \varepsilon)^{n / 4}$, as $\varepsilon \rightarrow 0$. On the other hand, the "natural" solution of unwinding the spiral one bar at a time fits nicely into the setup of mechanisms and the parametric linear program approach.

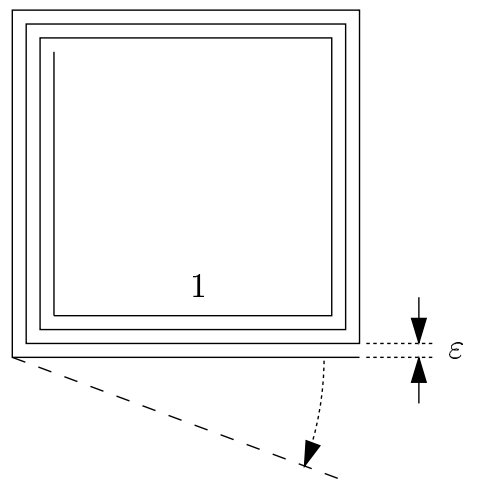

Figure 12: An arc that is numerically difficult to unfold.

Our proof has certain nonconstructive aspects: the direction $\mathbf{v}$ of movement is specified implicitly as the solution of an optimization problem, and the global motion arises as the solution of a differential equation. Both of these items are numerically well-understood, and our approach lends itself to a practical implementation. Indeed, we implemented our approach to produce animations such as Figure 1. However, this does not necessarily lead to a finite algorithm in the strict sense. The optimization problem (7-9), having an objective function which is rational, can in principle be solved exactly by solving the system $h(p, x, \lambda)=$ 
0 of algebraic equations as in Lemma 7. The differential equation cannot be solved explicitly, but it may be possible to bound the convergence and solve the differential equation up to a given error bound.

Because the motions of a mechanism are described by algebraic equations, Streinu's algorithm leads to a finite algorithm for a digital computer, at least in principle. It remains to be seen how a practical implementation competes with our approach; in any case, as an algorithm for a direct realization of the motion by a mechanical device, Streinu's algorithm appears attractive.

On the other hand, our nonlinear programming approach might be preferable because it produces a "canonical" movement. As a consequence of this, any symmetry of the starting configuration is preserved (see Corollary 2 in the next section).

\section{Additional Properties and Related Problems}

\subsection{Symmetry}

We show that the deformation that we have defined in Section 4 preserves any symmetries that the original configuration might have. We say that the arc-and-cycle set $A$ has some group $H$ of congruences of the plane as symmetry group if each element of $H$ permutes the vertices and edges of $A$.

Corollary 2 If an arc-and-cycle set has a symmetry group $H$, then there is a piecewisedifferentiable proper motion to an outer-convex configuration; the motion is expansive until the linkage becomes separated, and the symmetry group $H$ is preserved during the motion.

Proof: Because $A$ has finitely many vertices and edges, $H$ must be finite, so the Affine Fixed Point Theorem implies that there must be a point fixed by all elements of $H$. Let this point be the origin, and let $\mathbf{p}_{1}$ be any vertex of the configuration distinct from the origin. Consider the infinitesimal motion defined by the conditions (7), (8), and (9) but not (10). There is a unique solution $\mathbf{v}$ to this minimization problem. This solution must be symmetric with respect to the symmetry group $H$. If not, then the action of some element of $H$ takes $\mathbf{v}$ to a distinct solution, contradicting the uniqueness of the solution. There is now a unique infinitesimal rotation that we can add to $\mathbf{v}$ so that $\mathbf{p}_{1}$ and $\mathbf{v}_{1}$ are parallel. This still maintains the symmetry of the infinitesimal motion $\mathbf{v}$. Now it is clear that the limit exists as before in the proof of Theorem 1, and the symmetry of $H$ is preserved.

\subsection{Increasing Area}

A natural question is whether every expansive motion increases the area bounded by each polygonal cycle. The answer turns out to be yes, but the proof is difficult from elementary methods. A simple example that helps motivate why this problem is nontrivial is an obtuse triangle: if the base edge increases in length (as a strut) and the others remain the same length (as bars), then the area decreases. The cycle of bars in a polygonal cycle is therefore crucial but difficult to exploit except with our theory of expansive motions. 
First we show how to extend any given expansive infinitesimal motion to any point in the plane, which is of interest in its own right.

Lemma 11 Consider an infinitesimal motion $\mathbf{v}$ on points $\mathbf{p}_{1}, \ldots, \mathbf{p}_{n}$ in $\mathbb{R}^{d}$, and suppose that the motion is expansive, i.e., $\left(\mathbf{p}_{i}-\mathbf{p}_{j}\right) \cdot\left(\mathbf{v}_{i}-\mathbf{v}_{j}\right) \geq 0$ for all $i, j$. Then the infinitesimal motion $\mathbf{v}$ can be extended to another point $\mathbf{p}_{0}$ in $\mathbb{R}^{d}$ and remain expansive. Furthermore, the new expansiveness inequalities are all strict unless $\mathbf{p}_{0}$ is in the convex hull of a subset of points on which the original infinitesimal motion is trivial, i.e., describes a rigid motion.

Proof: We have two proofs of this statement: the first proof is a calculation and the second proof uses Helly's Theorem.

We first consider the case where we want to prove strict expansiveness. By the Farkas lemma, the desired inequalities $\left(\mathbf{v}_{0}-\mathbf{v}_{i}\right) \cdot\left(\mathbf{p}_{0}-\mathbf{p}_{i}\right)>0$, can be fulfilled by some unknown vector $\mathbf{v}_{0}$ if and only if the dual system

$$
\begin{aligned}
\sum_{i=1}^{n} \lambda_{i}\left(\mathbf{p}_{0}-\mathbf{p}_{i}\right) & =0 \\
\sum_{i=1}^{n} \lambda_{i} \mathbf{v}_{i} \cdot\left(\mathbf{p}_{0}-\mathbf{p}_{i}\right) & \geq 0 \\
\lambda_{i} & \geq 0
\end{aligned}
$$

has no solution except the trivial solution $\lambda \equiv 0$. In order to find a contradiction, suppose that a nontrivial solution $\lambda$ exists. Without loss of generality, we may assume

$$
\sum_{i=1}^{n} \lambda_{i}=1
$$

Then we get from (17) a representation of $\mathbf{p}_{0}$ as a convex combination

$$
\mathbf{p}_{0}=\sum_{i=1}^{n} \lambda_{i} \mathbf{p}_{i}
$$

Substituting this into (18) yields

$$
\sum_{i=1}^{n} \sum_{j=1}^{n} \lambda_{i} \lambda_{j}\left(\mathbf{v}_{i} \cdot \mathbf{p}_{j}\right)-\sum_{i=1}^{n} \lambda_{i}\left(\mathbf{v}_{i} \cdot \mathbf{p}_{i}\right) \geq 0 .
$$

On the other hand, multiplying the given inequalities

$$
\mathbf{v}_{i} \cdot \mathbf{p}_{i}-\mathbf{v}_{i} \cdot \mathbf{p}_{j}-\mathbf{v}_{j} \cdot \mathbf{p}_{i}+\mathbf{v}_{j} \cdot \mathbf{p}_{j} \geq 0
$$

by $-\lambda_{j} \lambda_{j} / 2$ and summing them over $i, j=1, \ldots, n$ (including the trivial cases for $i=j$ ) yields

$$
\sum_{i=1}^{n} \sum_{j=1}^{n} \lambda_{i} \lambda_{j}\left(\mathbf{v}_{i} \cdot \mathbf{p}_{j}\right)-\sum_{i=1}^{n} \lambda_{i}\left(\mathbf{v}_{i} \cdot \mathbf{p}_{i}\right) \leq 0 .
$$


By the assumption of the lemma, we have $\lambda_{i}>0$ in (19) for at least two points $\mathbf{p}_{i}$ and $\mathbf{p}_{j}$ whose distance expands strictly. This means that the corresponding strict inequality in (21) will hold in (22) too, a contradiction to (20). This finishes the case when $\mathbf{p}_{0}$ does not lie in the convex hull of some points which move rigidly.

In the other case, nonstrict expansiveness can be shown by a variation of the above argument. Alternatively, we can appeal to Lemma 2 (or its higher-dimensional extension) and let the point $\mathbf{p}_{0}$ move rigidly with the rigid point set in whose convex hull it lies. The resulting motion is expansive; the distance from $\mathbf{p}_{0}$ to the other points will expand strictly, with the obvious exception of the points with which it moves rigidly. This concludes the first proof of the lemma.

The other proof establishes a connection to tensegrity frameworks and is more intuitive. However, we have to deal with a few extra cases to reduce the statement of the lemma to the basic case that the points $\mathbf{p}_{1}, \ldots, \mathbf{p}_{n}$ form the vertices of a simplex that contains the point $\mathbf{p}_{0}$ in its interior.

We proceed by induction on the dimension $d$. There is nothing to prove in case $d=0$. So we assume the statement for $0, \ldots, d-1$ and $d \geq 1$. The desired inequalities $\left(\mathbf{v}_{0}-\mathbf{v}_{i}\right)$. $\left(\mathbf{p}_{0}-\mathbf{p}_{i}\right)>0$ define open half spaces

$$
H_{i}=\left\{\mathbf{v}_{0} \mid \mathbf{v}_{0} \cdot\left(\mathbf{p}_{0}-\mathbf{p}_{i}\right)>\mathbf{v}_{i} \cdot\left(\mathbf{p}_{0}-\mathbf{p}_{i}\right)\right\} .
$$

This finite collection of half spaces is nonempty precisely if every set of at most $d+1$ of them is nonempty by Helly's theorem [DGK63]. So we consider any subset of $k \leq d+1$ points $\mathbf{p}_{1}, \mathbf{p}_{2}, \ldots, \mathbf{p}_{k}$ of $\mathbf{p}$. If $\mathbf{p}_{0}$ is outside the convex hull $\sigma$ of these points, then simply choose $\mathbf{v}_{0}$ in a direction along a normal to a hyperplane separating $\mathbf{p}_{0}$ and $\sigma$, pointing away from $\sigma$. If the magnitude of $\mathbf{v}_{0}$ is large enough, then the desired inequalities will be satisfied.

If $\mathbf{p}_{0}$ lies in the convex hull of the given points, we first consider the "general" case that there are $k=d+1$ affine-independent points, forming the vertices of the $d$-dimensional simplex $\sigma$ in $\mathbb{R}^{d}$, and $\mathbf{p}_{0}$ is interior to $\sigma$. Suppose that the inequalities defined above do not have a solution. Then if we look at the complementary half-spaces defined by

$$
H_{i}^{-}=\left\{\mathbf{v}_{0} \mid \mathbf{v}_{0} \cdot\left(\mathbf{p}_{0}-\mathbf{p}_{i}\right) \leq \mathbf{v}_{i} \cdot\left(\mathbf{p}_{0}-\mathbf{p}_{i}\right)\right\},
$$

they do have a solution. Let $\mathbf{v}=\left(\mathbf{v}_{0}, \mathbf{v}_{1}, \mathbf{v}_{2}, \ldots, \mathbf{v}_{d+1}\right)$ be a solution to those new inequalities. Now $\mathbf{v}$ is an infinitesimal motion of the tensegrity that is obtained by having cables from $\mathbf{p}_{0}$ (whose lengths can only shrink) and the rest all struts as before. But it is easy to show that this tensegrity has no infinitesimal motion in $\mathbb{R}^{d}$. (For example, apply Theorem 5.2 (c) of [RW81] observing that the underlying bar framework has no non-trivial infinitesimal motion, and there must be a proper stress that is nonzero on all struts and cables. An explicit calculation of the proper stress is given in [BC99]. The calculation is similar to the calculations (19)-(22) in the first part of the proof, with $\lambda_{i} \lambda_{j}$ being interpreted as stress.) So $\mathbf{v}_{0}, \mathbf{v}_{1}, \mathbf{v}_{2}, \ldots, \mathbf{v}_{d+1}$ must be a trivial infinitesimal motion, which can only happen if the motion is trivial on all of $\sigma$.

There are two remaining cases:

(a) The points $\mathbf{p}_{1}, \mathbf{p}_{2}, \ldots, \mathbf{p}_{k}$ lie in a hyperplane $S$. This includes the cases when $k<d+1$ and when the points are affine-dependent. 
(b) There are $k=d+1$ points $\mathbf{p}_{1}, \mathbf{p}_{2}, \ldots, \mathbf{p}_{d+1}$ forming a $d$-dimensional simplex $\sigma$ in $\mathbb{R}^{d}$, and $\mathbf{p}_{0}$ lies on the boundary of $\sigma$.

In case (a), we know that $\mathbf{p}_{0} \in S$, because otherwise it would lie outside the convex hull $\sigma$. We decompose each $\mathbf{v}_{i}$ into a component $\mathbf{v}_{i}^{\|}$parallel to $S$ and a component $\mathbf{v}_{i}^{\perp}$ perpendicular to $S$. By the induction hypothesis, there is a vector $\mathbf{v}_{0}^{\|}$parallel to $S$ such that it together with the other vertices is infinitesimally expansive with respect to the projected $\mathbf{v}_{i}^{\|}$and hence the $\mathbf{v}_{i}$ themselves. It is strict unless $\mathbf{p}_{0}$ is in the convex hull of a subset of the points where the infinitesimal motion is trivial.

In case (b), there is a hyperplane $S$ containing $\mathbf{p}_{0}$ and all of the points $\mathbf{p}_{1}, \mathbf{p}_{2}, \ldots, \mathbf{p}_{d+1}$ except one, say $\mathbf{p}_{d+1}$. We apply the construction of case (a) to the points $\mathbf{p}_{1}, \mathbf{p}_{2}, \ldots, \mathbf{p}_{d}$, yielding a vector $\mathbf{v}_{0}^{\|}$which is infinitesimally expansive with respect to these vertices. To get expansiveness also for $\mathbf{p}_{d+1}$, we add to $\mathbf{v}_{0}^{\|}$a sufficiently large vector $\mathbf{v}_{0}^{\perp}$ perpendicular to $S$, pointing into the halfspace of $S$ which does not contain $\mathbf{p}_{d+1}$. Since the added vector $\mathbf{v}_{0}^{\perp}$ is perpendicular to $S$, this does not affect expansiveness with respect to the points $\mathbf{p}_{1}, \mathbf{p}_{2}, \ldots, \mathbf{p}_{d}$ in $S$.

Thus in any case we see that there is a strict solution to the inequalities for $\mathbf{p}_{0}, \mathbf{p}_{1}, \mathbf{p}_{2}, \ldots$, $\mathbf{p}_{d+1}$, or $\mathbf{p}_{0}$ is in the convex hull of points where the infinitesimal motion acts as bars for all surrounding points. This concludes the second proof of the lemma.

Now we apply this lemma to prove that the area of a polygonal cycle increases by any expansive motion. Using the lemma inductively, we can extend an expansive motion to any finite set of points. Specifically, we apply Lemma 11 to the vertices of an appropriately chosen triangulation of the region bounded by a polygonal cycle. (The triangulation introduces new vertices in addition to the vertices of the polygonal cycle.) The following result can be found in [BGR88]. (See also [BMR95, Epp97] for faster algorithms.)

Lemma 12 Any simple closed polygonal curve in the plane can be triangulated, introducing extra vertices, such that all the triangles are nonobtuse, i.e., every angle is at most $\pi / 2$.

There has been some interest in providing acute triangulations and subdivisions (in contrast to nonobtuse triangulations) of various planar polygonal objects. For example, the column of Martin Gardner [Gar60] (see also [Gar95] and [Man60]) asks for a dissection of a right triangle into acute triangles. but we do not know of a result guaranteeing an acute triangulation for a general polygon. Fortunately, the following is sufficient for the area-expanding property that we need:

Lemma 13 Let $\mathbf{v}=\left(\mathbf{v}_{1}, \mathbf{v}_{2}, \mathbf{v}_{3}\right)$ be an infinitesimal motion of a nonobtuse triangle $\mathbf{p}=$ $\left(\mathbf{p}_{1}, \mathbf{p}_{2}, \mathbf{p}_{3}\right)$ such that for $i \neq j$,

$$
\left(\mathbf{v}_{i}-\mathbf{v}_{j}\right) \cdot\left(\mathbf{p}_{i}-\mathbf{p}_{j}\right) \geq 0 .
$$

Then the infinitesimal change in the area of triangle $\mathbf{p}$ is always nonnegative. Furthermore, the infinitesimal change in the area is positive except in the following two cases:

(a) The infinitesimal flex $\mathbf{v}$ is trivial, i.e., no inequality in (23) is strict. 
Proof: Let the lengths of the sides of the triangle be denoted by $a, b, c$, and let the area of the triangle be denoted by $A$. If we differentiate Heron's formula

$$
16 A^{2}=2\left(a^{2} b^{2}+a^{2} c^{2}+b^{2} c^{2}\right)-\left(a^{4}+b^{4}+c^{4}\right)
$$

and rearrange terms, denoting derivatives by $a^{\prime}, b^{\prime}, c^{\prime}, A^{\prime}$, we get

$$
8 A A^{\prime}=\left(b^{2}+c^{2}-a^{2}\right) a a^{\prime}+\left(a^{2}+c^{2}-b^{2}\right) b b^{\prime}+\left(a^{2}+b^{2}-c^{2}\right) c c^{\prime} .
$$

We can regard $a a^{\prime}, b b^{\prime}, c c^{\prime}$ as the left hand side of (23). Each of the terms in parentheses in (24) is nonnegative because $\mathbf{p}$ is nonobtuse. Thus $A^{\prime} \geq 0$.

If $\mathbf{p}$ is an acute triangle and at least one of $a^{\prime}>0$ or $b^{\prime}>0$ or $c^{\prime}>0$, then (24) is positive, and thus $A^{\prime}>0$. Suppose $\mathbf{p}$ is a right triangle and $c$ represents the length of the hypotenuse. If at least one of $a^{\prime}>0$ or $b^{\prime}>0$, then (24) is positive, so $A^{\prime}>0$.

Note that with a right triangle it is possible for the first derivative of the length of the hypotenuse to be positive while the first derivative of the length of the two legs is 0 , and in this case the first derivative of the area will still be 0 . This is the reason for the condition on the legs of the triangle.

Theorem 7 Any smooth expansive noncongruent motion of a simple closed polygonal curve $C$ in the plane, fixing the lengths of its edges, must increase the area of the interior of $C$ during the motion.

Proof: Consider the vector field $\mathbf{v}_{t}, 0 \leq t \leq 1$ defined as the derivative at each vertex of $C$ at time $t$. Apply Lemma 12 to find a triangulation $T$ of the area bounded by $C$ with all triangles nonobtuse. Apply Lemma 11 to extend the vector field to the vertices of $T$.

To get a strictly increasing area, we have to show that the triangulation $T$ has an acute triangle with an edge interior to $C$, or a right triangle with a leg interior to $C$. Otherwise, $T$ would be a single triangle, or it would exclusively consist of right triangles with both legs on $C$, hence it would be a convex quadrilateral with two opposite corners having right angles. These cases are excluded because a triangle or a convex quadrilateral (or any convex polygon) does not have an expansive noncongruent motion.

So we have established that $T$ must have an acute triangle with an edge interior to $C$ or a right triangle with a leg interior to $C$. Because the motion is expansive and the derivative of at least one of those lengths must be positive for all but a finite number of times, the derivative of the area of at least one of those triangles must be strictly positive, and they all are nonnegative by Lemma 13. So the derivative of the area bounded by $C$ must be positive for all but a finite number of times $0 \leq t \leq 1$. Thus the area must strictly increase.

\subsection{Topology of Configuration Spaces}

It is natural to ask more about the structure of the configuration space of an arc-and-cycle set. Let $X(G, L)$ denote the space of all configurations of embeddings in the plane of of a 
bar graph $G$ consisting of a finite number arcs and cycles, without self-intersections, where the edge lengths are determined by $L=\left(\ldots, \ell_{i j}, \ldots\right)$. This inherits a natural topology from considering all the coordinates of all the vertices as part of a large dimensional Euclidean space. Let $X_{0}(G, L) \subset X(G, L)$ denote the subspace of outer-convex configurations. We assume that $L$ is chosen so that there is at least one realization in the plane. We mention some results without proof.

Theorem 8 The space of outer-convex realizations $X_{0}(G)$ is a strong deformation retract of $X(G)$.

The main point to remember is that the limit in Theorem 1 depends continuously on the initial starting configuration. The following is a natural consequence of Theorem 8 .

Corollary 3 If the underlying graph $G$ is a single arc or a single cycle, then $X(G, L)$ modulo congruences (including orientation reversing ones) is contractible.

Here the main task is to show that the space of convex realizations is contractible.

It is interesting to compare $X(G, L)$, as we have defined it, to the space of realizations of an arc or cycle in the plane with fixed edge lengths, but where crossings are allowed. See e.g. [LW95, KM99, KM95a, KM95b, KM96] for results about this space.

\subsection{Open Problems}

Another direction is to explore what happens when the arc-and-cycle set is allowed to touch but not cross:

Conjecture 1 If $G$ is a single arc or a single cycle, then the closure of $X(G, L)$ modulo congruences is contractible.

We conjecture that motions can be realized by a sequence of relatively simple motions:

Conjecture 2 If $A$ is an arc-and-cycle set in the plane, then there is a motion that takes it to an outer-convex configuration, by a finite sequence of motions, where each motion changes at most four vertex angles.

It also remains open precisely how many such moves are needed.

\section{Acknowledgments}

The second author's main interest in this research was initiated at the International Workshop on Wrapping and Folding organized by Anna Lubiw and Sue Whitesides at the Bellairs Research Institute of McGill University in February 1998. At this workshop, a bond between several linkage openers began: Therese Biedl, Martin Demaine, Hazel Everett, Sylvain Lazard, Anna Lubiw, Joseph O’Rourke, Mark Overmars, Steven Robbins, Ileana Streinu, 
Godfried Toussaint, Sue Whitesides, and the second author. The group of "linkage unlockers" mentioned in the introduction soon grew to include Sándor Fekete, Joseph Mitchell, and the authors. Many other people in various fields have worked on the problem.

One of the key meetings that started this paper is the Monte Verità Conference on Discrete and Computational Geometry in Ascona, Switzerland, organized by Jacob Goodman, Richard Pollack, and Emo Welzl in June 1999. Some of the key ideas were initially discussed at this conference. The proof of the main result was essentially conceived at the 4th Geometry Festival, an international workshop on Discrete Geometry and Rigidity, in Budapest, Hungary, organized by András Bezdek, Károly Bezdek, Károly Böröczky, and the first author, in November 1999. We thank the organizers of these workshops, in particular for the opportunity to meet and work together. Work was continued during a visit of Erik Demaine in Berlin in December 1999, sponsored by the graduate program Graduiertenkolleg "Algorithmische Diskrete Mathematik" of the Deutsche Forschungsgemeinschaft, grant GRK 219/3.

We thank Walter Whiteley for helpful discussions about rigidity theory. We thank Bernd Kummer for discussions about stability in optimization problems. We thank Joseph O'Rourke for our discussions about the increasing-area property (Section 5.2). Finally, we thank Therese Biedl, Anna Lubiw, Ian Munro, and the anonymous referees for many helpful comments on the manuscript.

\section{References}

[AR78] L. Asimow and B. Roth. The rigidity of graphs. Transactions of the American Mathematical Society, 245:279-289, 1978.

[AR79] L. Asimow and B. Roth. The rigidity of graphs. II. Journal of Mathematical Analysis and Applications, 68(1):171-190, 1979.

[BGR88] Brenda S. Baker, Eric Grosse, and Conor S. Rafferty. Nonobtuse triangulation of polygons. Discrete \& Computational Geometry, 3(2):147-168, 1988.

[BMR95] Marshall Bern, Scott Mitchell, and Jim Ruppert. Linear-size nonobtuse triangulation of polygons. Discrete \& Computational Geometry, 14(4):411-428, 1995.

[BC99] Károly Bezdek and Robert Connelly. Two-distance preserving functions from Euclidean space. Periodica Mathematica Hungarica, 39(1-3):185-200, 1999.

$\left[\mathrm{BDD}^{+} 01\right]$ T. Biedl, E. Demaine, M. Demaine, S. Lazard, A. Lubiw, J. O’Rourke, M. Overmars, S. Robbins, I. Streinu, G. Toussaint, and S. Whitesides. Locked and unlocked polygonal chains in three dimensions. Discrete 89 Computational Geometry, 26(3):283-287, October 2001. The full version is Technical Report 060, Smith College, 1999, and arXiv:cs.CG/9910009, http://www.arXiv.org/abs/ Cs.CG/9910009.

$\left[\mathrm{BDD}^{+} 98\right]$ Therese Biedl, Erik Demaine, Martin Demaine, Sylvain Lazard, Anna Lubiw, Joseph O'Rourke, Steve Robbins, Ileana Streinu, Godfried Toussaint, and Sue 
Whitesides. On reconfiguring tree linkages: Trees can lock. In Proceedings of the 10th Canadian Conference on Computational Geometry, Montréal, Canada, August 1998. http://cgm.cs.mcgill.ca/cccg98/proceedings/cccg98-biedlreconfiguring.ps.gz.

$\left[\mathrm{BDL}^{+} 99\right]$ Therese C. Biedl, Erik D. Demaine, Sylvain Lazard, Steven M. Robbins, and Michael A. Soss. Convexifying monotone polygons. In Proceedings of the 10th Annual International Symposium on Algorithms and Computation, volume 1741 of Lecture Notes in Computer Science, pages 415-424, Chennai, India, December 1999. An expanded version is Technical Report CS-99-03, Department of Computer Science, University of Waterloo, 1999.

[BDST99] Therese C. Biedl, Erik D. Demaine, Michael A. Soss, and Godfried T. Toussaint. Straightening visible chains under constraints. Technical Report CS-99-08, Department of Computer Science, University of Waterloo, 1999.

[BS74] James H. Bigelow and Norman Z. Shapiro. Implicit function theorems for mathematical programming and for systems of inequalities. Mathematical Programming, 6:141-156, 1974.

[CJ98] Jason Cantarella and Heather Johnston. Nontrivial embeddings of polygonal intervals and unknots in 3-space. Journal of Knot Theory and Its Ramifications, 7(8):1027-1039, 1998.

[Cau13] A. L. Cauchy. Deuxième mémoire sur les polygones et polyèdres (Second memoire on polygons and polyhedra). Journal de l'École Polytechnique, 9:87-98, May 1813.

[CO99] Roxana Cocan and Joseph O'Rourke. Polygonal chains cannot lock in 4D. In Proceedings of the 11th Canadian Conference on Computational Geometry, Vancouver, Canada, August 1999. http://www.cs.ubc.ca/conferences/CCCG/elec_ proc/c17.ps.gz.

[CO01] Roxana Cocan and Joseph O’Rourke. Polygonal chains cannot lock in 4D. Technical Report 063, Smith College, February 2001. http: //www.arXiv.org/abs / cs.CG/9908005.

[Con80] Robert Connelly. The rigidity of certain cabled frameworks and the second-order rigidity of arbitrarily triangulated convex surfaces. Advances in Mathematics, $37(3): 272-299,1980$.

[Con82] Robert Connelly. Rigidity and energy. Inventiones Mathematicae, 66(1):11-33, 1982.

[Con93] Robert Connelly. Rigidity. In Handbook of Convex Geometry, volume A, pages 223-271. North-Holland, Amsterdam, 1993. 
[CDR00] Robert Connelly, Erik D. Demaine, and Günter Rote. Straightening polygonal arcs and convexifying polygonal cycles. In Proceedings of the 41st Annual Symposium on Foundations of Computer Science, pages 432-442, Redondo Beach, California, November 2000.

[CDR02] Robert Connelly, Erik D. Demaine, and Günter Rote. Infinitesimally locked selftouching linkages with applications to locked trees. Manuscript in preparation, 2002 .

[CW96] Robert Connelly and Walter Whiteley. Second-order rigidity and prestress tensegrity frameworks. SIAM Journal on Discrete Mathematics, 9(3):453-491, 1996.

[CW82] Henry Crapo and Walter Whiteley. Statics of frameworks and motions of panel structures, a projective geometric introduction (with a French translation). Structural Topology, 6:43-82, 1982.

[CW93] Henry Crapo and Walter Whiteley. Autocontraintes planes et polyèdres projetés. I. Le motif de base [Plane self stresses and projected polyhedra. I. The basic pattern]. Structural Topology, 20:55-78, 1993.

[CW94] Henry Crapo and Walter Whiteley. Spaces of stresses, projections and parallel drawings for spherical polyhedra. Beiträge zur Algebra und Geometrie, 35(2):259281, 1994.

[Cro97] Peter R. Cromwell. Equality, rigidity, and flexibility. In Polyhedra, chapter 6, pages 219-247. Cambridge University Press, 1997.

[DGK63] Ludwig Danzer, Branko Grünbaum, and Victor Klee. Helly's theorem and its relatives. In Proc. Sympos. Pure Math., volume VII, pages 101-180. American Mathematical Society, 1963.

[Epp97] David Eppstein. Faster circle packing with application to nonobtuse triangulation. International Journal of Computational Geometry and Applications, $7(5): 485-491,1997$.

[Erd35] Paul Erdős. Problem 3763. American Mathematical Monthly, 42:627, 1935.

[ELR $\left.{ }^{+} 98\right]$ H. Everett, S. Lazard, S. Robbins, H. Schröder, and S. Whitesides. Convexifying star-shaped polygons. In Proceedings of the 10th Canadian Conference on Computational Geometry, Montréal, Canada, August 1998. http://cgm.cs.mcgill. ca/cccg98/proceedings/cccg98-everett-convexifying.ps.gz.

[Eve93] Brain S. Everitt. Cluster Analysis. Edward Arnold (London) and Halsted Press (New York), third edition, 1993.

[Fia76] Anthony V. Fiacco. Sensitivity analysis for nonlinear programming using penalty methods. Mathematical Programming, 10:287-311, 1976.

[FK97] Maxim D. Frank-Kamenetskii. Unravelling DNA. Addison-Wesley, 1997. 
[Gar60] Martin Gardner. Mathematical games. Scientific American, 202, 1960. The problem is posed in the February issue (number 202) on page 150. A dissection into seven parts appears in the March issue (number 3), pages 177-178.

[Gar95] Martin Gardner. New Mathematical Diversions, pages 34 and 39-42. Mathematical Association of America, Washington, D.C., 1995.

[Glu74] Herman Gluck. Almost all simply connected closed surfaces are rigid. In Proceedings of the Geometric Topology Conference, volume 438 of Lecture Notes in Mathematics, pages 225-239, Park City, Utah, February 1974.

[GSS93] Jack Graver, Brigitte Servatius, and Herman Servatius. Combinatorial rigidity. American Mathematical Society, Providence, 1993.

[GCK91] U. Grenander, Y. Chow, and D. M. Keenan. Hands: A Pattern Theoretic Study of Biological Shapes. Springer-Verlag, 1991.

[Grü95] Branko Grünbaum. How to convexify a polygon. Geombinatorics, 5:24-30, July 1995.

[HJ97] Pierre Hansen and Brigitte Jaumard. Cluster analysis and mathematical programming. Mathematical Programming, 79B:191-215, 1997.

[HK92] John E. Hopcroft and Peter J. Kahn. A paradigm for robust geometric algorithms. Algorithmica, 7(4):339-380, 1992.

[KM95a] Michael Kapovich and John Millson. On the moduli space of polygons in the Euclidean plane. Journal of Differential Geometry, 42(1):133-164, 1995.

[KM95b] Michael Kapovich and John Millson. On the moduli space of polygons in the Euclidean plane. Journal of Differential Geometry, 42(2):430-464, 1995.

[KM96] Michael Kapovich and John J. Millson. The symplectic geometry of polygons in Euclidean space. Journal of Differential Geometry, 44(3):479-513, 1996.

[KM99] Michael Kapovich and John J. Millson. Moduli spaces of linkages and arrangements. In Advances in Geometry, volume 172 of Progress in Mathematics, pages 237-270. Birkhäuser, Boston, 1999.

[Kir97] Rob Kirby. Problems in low-dimensional topology. In Geometric Topology: Proceedings of the 1993 Georgia International Topology Conference, volume 2.2 of AMS/IP Studies in Advanced Mathematics, pages 35-473. American Mathematical Society and International Press, 1997. http://www.math.berkeley.edu/ 〜kirby/problems.ps.gz.

[LW95] W. J. Lenhart and S. H. Whitesides. Reconfiguring closed polygonal chains in Euclidean $d$-space. Discrete \& Computational Geometry, 13:123-140, 1995. 
[LW91] William J. Lenhart and Sue H. Whitesides. Turning a polygon inside-out. In Proceedings of the 3rd Canadian Conference on Computational Geometry, pages 66-69, Vancouver, Canada, August 1991.

[LW93] William J. Lenhart and Sue H. Whitesides. Reconfiguring simple polygons. Technical Report SOCS-93.3, School of Computer Science, McGill University, March 1993.

[MOS90] N. Madras, A. Orlitsky, and L. A. Shepp. Monte Carlo generation of self-avoiding walks with fixed endpoints and fixed length. Journal of Statistical Physics, 58(12):159-183, 1990.

[Man60] Wallace Manheimer. Solution to problem E 1406: Dissecting an obtuse triangle into acute triangles. American Mathematical Monthly, 67:923, November 1960.

[McM79] Frank M. McMillan. The Chain Straighteners. MacMillan Press, London, 1979.

[Mil94] Kenneth C. Millett. Knotting of regular polygons in 3-space. Journal of Knot Theory and its Ramifications, 3(3):263-278, 1994.

[Nag39] Béla de Sz. Nagy. Solution to problem 3763. American Mathematical Monthly, 46:176-177, March 1939.

[O'R98] Joseph O'Rourke. Folding and unfolding in computational geometry. In Revised Papers from the Japan Conference on Discrete and Computational Geometry, volume 1763 of Lecture Notes in Computer Science, pages 258-266, Tokyo, Japan, December 1998.

[RSS02] Günter Rote, Francisco Santos, and Ileana Streinu. Expansive motions and the polytope of pointed pseudo-triangulations. Manuscript, 2001, submitted for publication.

[RW81] B. Roth and W. Whiteley. Tensegrity frameworks. Transactions of the American Mathematical Society, 265(2):419-446, 1981.

[Sal73] G. T. Sallee. Stretching chords of space curves. Geometriae Dedicata, 2:311-315, 1973.

[SZ67] I. J. Schoenberg and S. K. Zaremba. Cauchy's lemma concerning convex polygons. Canadian Journal of Mathematics, 19(4):1062-1071, 1967.

[SW88] C. E. Soteros and S. G. Whittington. Polygons and stars in a slit geometry. Journal of Physics A: Mathematical and General Physics, 21:L857-861, 1988.

[SG72] S. D. Stellman and P. J. Gans. Efficient computer simulation of polymer conformation. I. Geometric properties of the hard-sphere model. Macromolecules, 5:516-526, 1972. 
[Str00] Ileana Streinu. A combinatorial approach to planar non-colliding robot arm motion planning. In Proceedings of the 41st Annual Symposium on Foundations of Computer Science, pages 443-453, Redondo Beach, California, November 2000.

[Tou99] Godfried Toussaint. The Erdős-Nagy theorem and its ramifications. In Proceedings of the 11th Canadian Conference on Computational Geometry, Vancouver, Canada, August 1999. http://www.cs.ubc.ca/conferences/CCCG/elec_proc/ fp19.ps.gz.

[Wal96] Wolfgang Walter. Gewöhnliche Differentialgleichungen. Springer-Verlag, Berlin, 6th edition, 1996. English translation: Ordinary differential equations, Springer, New York, 1998.

[Weg93] Bernd Wegner. Partial inflation of closed polygons in the plane. Beiträge zur Algebra und Geometrie, 34(1):77-85, 1993.

[Weg96] Bernd Wegner. Chord-stretching convexifications of spherical polygons. In Proceedings of the Topology and Geometry Day, volume 10 of Textos de Matemática (Texts in Mathematics), Series B, pages 42-49, Coimbra, May 1996.

[Whi82] Walter Whiteley. Motions and stresses of projected polyhedra (with a French translation). Structural Topology, 7:13-38, 1982.

[Whi84a] Walter Whiteley. Infinitesimally rigid polyhedra. I. statics of frameworks. Transactions of the American Mathematical Society, 285(2):431-465, 1984.

[Whi84b] Walter Whiteley. The projective geometry of rigid frameworks. In Finite geometries, volume 103 of Lecture Notes in Pure and Applied Mathematics, pages 353-370, Winnipeg, Manitoba, 1984.

[Whi87] Walter Whiteley. Rigidity and polarity. I. Statics of sheet structures. Geometriae Dedicata, 22(3):329-362, 1987.

[Whi88] Walter Whiteley. Infinitesimally rigid polyhedra. II. Modified spherical frameworks. Transactions of the American Mathematical Society, 306(1):115-139, 1988.

[Whi92a] Walter Whiteley. Matroids and rigid structures. In Neil White, editor, Matroid applications, volume 40 of Encyclopedia of Mathematics and its Applications, pages 1-52. Cambridge University Press, Cambridge, 1992.

[Whi92b] Sue Whitesides. Algorithmic issues in the geometry of planar linkage movement. Australian Computer Journal, 24(2):42-50, May 1992.

[Whi83] S. G. Whittington. Self-avoiding walks with geometrical constraints. Journal of Statistical Physics, 30(2):449-456, 1983. 


\section{A Appendix: Expansive Motion for Several Compo- nents}

37:02

37:03

$37: 04$

$37: 05$

$37: 06$

$37: 07$

$37: 08$

37:09

$37: 10$

$37: 11$

$37: 12$

$37: 13$

$37: 14$

$37: 15$

$37: 16$

$37: 17$

$37: 18$

$37: 19$

$37: 20$

$37: 21$

$37: 22$

$37: 23$

$37: 24$

$37: 25$

$37: 26$

$37: 27$

$37: 28$

$37: 29$

$37: 30$

$37: 31$

$37: 32$

$37: 33$

$37: 34$

$37: 35$

The motion described so far is expansive in each component, but may not expand the distance between vertices on different components once those components separate. In this final section we prove the following stronger version of Theorem 1 that guarantees a strictly expansive motion for multiple components even after the components separate. This result is attractive because it allows us to specify a canonical motion all the way to completion. Unfortunately, the proof is long.

Theorem 9 Every arc-and-cycle set has a piecewise differentiable proper motion to an outer-convex configuration that is strictly expansive throughout the motion.

Proof: As mentioned in Section 4.2, the motion is defined in the same way as for Theorem 1, except that the explicit pinning constraint (10) is removed from the definition of $\mathbf{v}=f(\mathbf{p})$.

As in the case of Theorem 1, we know that the initial value problem (12) has a unique maximal solution $\mathbf{p}(t), 0 \leq t<T$, that cannot be extended beyond $T$, and we have $T<\infty$. We have to show that, for $t \rightarrow T$, the configuration $\mathbf{p}(t)$ converges to a configuration on the boundary of $U$. From the Boundedness Lemma (Lemma 10 in Section 4.2), whose proof is given below, we conclude that the monotone limit

$$
K_{i}:=\lim _{t \rightarrow T} \int_{0}^{t}\left\|\mathbf{v}_{i}\right\| d t
$$

exists. Therefore, for all $t_{2} \geq t_{1} \geq t_{0}$, we have

$$
\left\|\mathbf{p}_{i}\left(t_{2}\right)-\mathbf{p}_{i}\left(t_{1}\right)\right\| \leq \int_{t_{1}}^{t_{2}}\left\|\mathbf{v}_{i}(t)\right\| d t \leq K_{i}-\int_{0}^{t_{0}}\left\|\mathbf{v}_{i}(t)\right\| d t .
$$

The last bound goes to zero as $t_{0} \rightarrow T$, and therefore $\mathbf{p}_{i}(t)$ converges. The limit configuration must lie on the boundary of the domain $U$ because otherwise the solution of (12) could be extended beyond $T$.

The rest of this appendix proves the only remaining aspect: the Boundedness Lemma (Lemma 10 in Section 4.2). That is, we would like to show that, under the expansive motion given by the optimization problem (7-9), the motion of individual points is limited.

Before embarking on the proof, let us consider a possibly difficult situation, which the proof has to handle. This example may aid the reader in appreciating the arguments of the proof.

We know that the distances change monotonically, and therefore their total change is bounded if the distances remain bounded. However, it is conceivable that the points move back and forth without increasing the distances too much. Consider three collinear points $\mathbf{p}_{1}, \mathbf{p}$ and $\mathbf{p}_{2}$ on a horizontal line; see Figure 13 . If the point $\mathbf{p}$ wiggles vertically up and down with a small and decreasing amplitude, one can maintain an expansive motion where all distances remain bounded and yet $\mathbf{p}$ moves on a path of infinite length. More precisely, we move $\mathbf{p}$ repeatedly up and down, each time returning to its original location, with the 


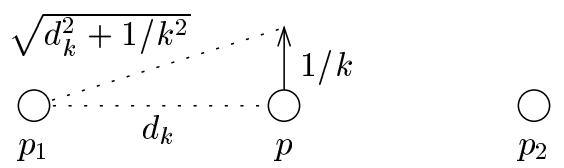

Figure 13: Wiggling point $\mathbf{p}$ vertically up and down by harmonic distances allows $\mathbf{p}$ to travel infinitely while pushing $\mathbf{p}_{1}$ and $\mathbf{p}_{2}$ away by only a bounded amount.

$38: 01$

$38: 02$

$38: 03$

$38: 04$

$38: 05$

$38: 06$

$38: 07$

$38: 08$

$38: 09$

$38: 10$

amount of vertical motion decreasing harmonically: 1, 1/2,1/3, 1/4, etc. Suppose the distance between $\mathbf{p}$ and both $\mathbf{p}_{1}$ and $\mathbf{p}_{2}$ is initially $d_{1}=1$, and after $k-1$ wiggles is $d_{k} \geq 1$. Then when $\mathbf{p}$ moves up $1 / k$ units, the distance between $\mathbf{p}$ and $\mathbf{p}_{i}$ increases from $d_{k}$ to

$$
d_{k+1}=\sqrt{d_{k}^{2}+1 / k^{2}}=d_{k}+\frac{1}{2 d_{k} k^{2}}+O\left(\frac{1}{d_{k}^{3} k^{4}}\right) \leq d_{k}+\frac{1}{k^{2}}+O\left(\frac{1}{k^{4}}\right) .
$$

When $\mathbf{p}$ moves back down $1 / k$ units, we move $\mathbf{p}_{1}$ left and move $\mathbf{p}_{2}$ right by this amount, to keep the motion expansive. After infinitely many wiggles, the distance between $\mathbf{p}$ and $\mathbf{p}_{i}$ is at most

$$
\sum_{k=1}^{\infty}\left(\sqrt{d_{k}^{2}+1 / k^{2}}-d_{k}\right) \leq \sum_{k=1}^{\infty}\left(\frac{1}{k^{2}}+O\left(\frac{1}{k^{4}}\right)\right)=O(1)
$$

while the total motion of $\mathbf{p}$ is

$$
\sum_{k=1}^{\infty} \frac{1}{k}=\infty
$$

This conceivable situation must be avoided.

We may get arbitrarily close to this situation if all points are very nearly collinear. We can however get a quantitative estimate of the motion by placing bounds on the minimum width and on the diameter of the point set. If the angles in the triangle $\mathbf{p}_{1} \mathbf{p} \mathbf{p}_{2}$ are not arbitrarily small, the motion of $\mathbf{p}$ relative to $\mathbf{p}_{1}$ and $\mathbf{p}_{2}$ can be bounded in terms of the increase of pairwise distances.

\section{A.1 Bounding the motion in terms of angles and distance changes}

We give two geometric lemmas that allow us to bound the motions in terms of quantities that can only change monotonically.

Lemma 14 Let $\mathbf{p}_{1}, \ldots, \mathbf{p}_{n}$ be the sequence of vertices of a component with the edges of fixed length $\left\|\mathbf{p}_{j}-\mathbf{p}_{j+1}\right\|=\ell_{j, j+1}$ for $j=1, \ldots, n-1$. (These constraints do not include the edge from $\mathbf{p}_{n}$ to $\mathbf{p}_{1}$ in the case of a polygonal cycle.) Say $\mathbf{p}_{1}$ and $\mathbf{p}_{2}$ are pinned, i.e., $\mathbf{v}_{1}=\mathbf{v}_{2}=0$. Let $\theta_{j}$ be the internal angle at vertex $j\left(0<\theta_{j}<\pi\right.$; we disregard the orientation of the angles). For any motion that preserves the lengths $\ell_{j, j+1}$, let $\theta_{j}^{\prime}$ denote the derivative of $\theta_{j}$ with respect to time, and let $\mathbf{v}_{k}$ denote the derivative of $\mathbf{p}_{k}$ with respect to time. Then, for $k=2, \ldots, n$,

$$
\left\|\mathbf{v}_{k}\right\| \leq\left(\ell_{1,2}+\cdots+\ell_{k-1, k}\right) \cdot\left(\theta_{2}^{\prime}+\cdots+\theta_{k-1}^{\prime}\right)
$$


39:06

Proof: Using complex notation,

$$
\frac{\mathbf{p}_{j+1}-\mathbf{p}_{j}}{\ell_{j, j+1}}=\frac{\mathbf{p}_{j-1}-\mathbf{p}_{j}}{\ell_{j-1, j}} \cdot \exp \left( \pm \theta_{j} i\right) .
$$

Taking derivatives gives

$$
\frac{\mathbf{v}_{j+1}-\mathbf{v}_{j}}{\ell_{j, j+1}}=\frac{\mathbf{v}_{j-1}-\mathbf{v}_{j}}{\ell_{j-1, j}} \cdot \exp \left( \pm \theta_{j} i\right)+\frac{\mathbf{p}_{j-1}-\mathbf{p}_{j}}{\ell_{j-1, j}} \cdot \exp \left( \pm \theta_{j} i\right) \cdot\left( \pm \theta_{j}^{\prime} i\right)
$$

Taking the norm of both sides and using the triangle inequality yields

$$
\frac{\left\|\mathbf{v}_{j+1}-\mathbf{v}_{j}\right\|}{\ell_{j, j+1}} \leq \frac{\left\|\mathbf{v}_{j-1}-\mathbf{v}_{j}\right\|}{\ell_{j-1, j}} \cdot \underbrace{\left|\exp \left( \pm \theta_{j} i\right)\right|}_{=1}+\underbrace{\frac{\left\|\mathbf{p}_{j-1}-\mathbf{p}_{j}\right\|}{\ell_{j-1, j}}}_{=1} \cdot \underbrace{\left|\exp \left( \pm \theta_{j} i\right)\right|}_{=1} \cdot\left|\theta_{j}^{\prime} i\right|=\frac{\left\|\mathbf{v}_{j-1}-\mathbf{v}_{j}\right\|}{\ell_{j-1, j}}+\left|\theta_{j}^{\prime}\right| .
$$

Applying this fact recursively,

$$
\frac{\left\|\mathbf{v}_{j+1}-\mathbf{v}_{j}\right\|}{\ell_{j, j+1}} \leq \frac{\left\|\mathbf{v}_{j-1}-\mathbf{v}_{j}\right\|}{\ell_{j-1, j}}+\left|\theta_{j}^{\prime}\right| \leq \cdots \leq\left|\theta_{2}^{\prime}\right|+\cdots+\left|\theta_{j}^{\prime}\right|
$$

and thus

$$
\left\|\mathbf{v}_{j+1}-\mathbf{v}_{j}\right\| \leq \ell_{j, j+1} \cdot\left(\left|\theta_{2}^{\prime}\right|+\cdots+\left|\theta_{j}^{\prime}\right|\right) \leq \ell_{j, j+1} \cdot\left(\left|\theta_{2}^{\prime}\right|+\cdots+\left|\theta_{k-1}^{\prime}\right|\right),
$$

for $j \leq k$. Applying induction we obtain the desired result.

The above lemma bounds the motion for a single component. For disconnected components, we want to bound the speed of motion $\mathbf{v}$ in terms of distance changes. However, it is always possible to move the point set as a whole without changing any distances at all. Therefore we will consider a modified motion in which one vertex $\mathbf{p}_{1}$ is fixed ("pinned"). Another vertex $\mathbf{p}_{2}$ remains on a fixed line through $\mathbf{p}_{1}$, preventing the complete set from spinning. This modification corresponds to adding a rigid motion of the point set $\mathbf{p}$ to the given velocities $\mathbf{v}$. It does not change the lengths $\ell_{i j}$ or their derivatives, $\left(\mathbf{v}_{i}-\mathbf{v}_{j}\right) \cdot\left(\mathbf{p}_{i}-\mathbf{p}_{j}\right)$.

The width of a set of points is the minimum width of a strip (infinitely long rectangle) containing the set.

Lemma 15 Consider a set $\mathbf{p}_{1}, \ldots, \mathbf{p}_{n}$ of $n \geq 3$ points with distances $\ell_{i j}=\left\|\mathbf{p}_{i}-\mathbf{p}_{j}\right\|$, moving with velocities $\mathbf{v}_{i}=\mathbf{p}_{i}^{\prime}$ under an expansive motion: $\ell_{i j}^{\prime} \geq 0$. Denote the initial width by $w$. Without loss of generality, we assume that the diameter $D$ is equal to $\ell_{12}$. We normalize the motion by pinning $\mathbf{p}_{1}$ and the direction of the axis from $\mathbf{p}_{1}$ to $\mathbf{p}_{2}$. In other words, we assume that $\mathbf{v}_{1}=0$ and that $\mathbf{v}_{2}$ is parallel to $\mathbf{p}_{2}-\mathbf{p}_{1}$. Then, for every $k \in\{1, \ldots, n\}$,

$$
\left\|\mathbf{v}_{k}\right\| \leq C_{0} \cdot \sum_{i<j} \ell_{i j}^{\prime}
$$

where $C_{0}=666 D^{3} / w^{3}$.

The proof of this lemma is tedious, and delayed to Section A.5 at the end. 


\section{A.2 Constant Quantities}

40:02

We define the following constants. We denote by $D_{0}$ the maximum sum of the bar lengths in a single component of the arc-and-cycle set. This is an upper bound on the diameter of a component. As we shall see, $D_{0} 6^{n-1}$, denoted henceforth as $D_{\max }$, is an upper bound on the distance between two points in the same cluster of components (a notion defined in the next subsection). Let $m_{0}\left(0<m_{0} \leq 1\right)$ be a lower bound on the distance between any two different components throughout the motion (achieved either as a distance between two vertices or as a distance from a vertex to an edge). Let $m_{1}\left(0<m_{1} \leq 1\right)$ be a lower bound on the distance between any two vertices (in the same component or in different components) throughout the motion. Because these distances can only increase during the expansive motion, we just need to choose $m_{0}$ and $m_{1}$ smaller than the minimum distance between two different components or between two vertices in the initial configuration.

The constant $C=5328 D_{\max }^{3} / m_{0}^{3}=8 \cdot 666 D_{\max }^{3} / m_{0}^{3}$ will play an important role in Lemma 19 and subsequently.

\section{A.3 Combining components into clusters}

In the proof we distinguish between components that are "close" to each other or even entangled within each other and components that are separated. Namely, we call two nonempty sets of points $A$ and $B$ well-separated if they can be separated by a line, and moreover, the two inner common tangents separating $A$ and $B$ form an angle less than $60^{\circ}$. See Figure 14(a).
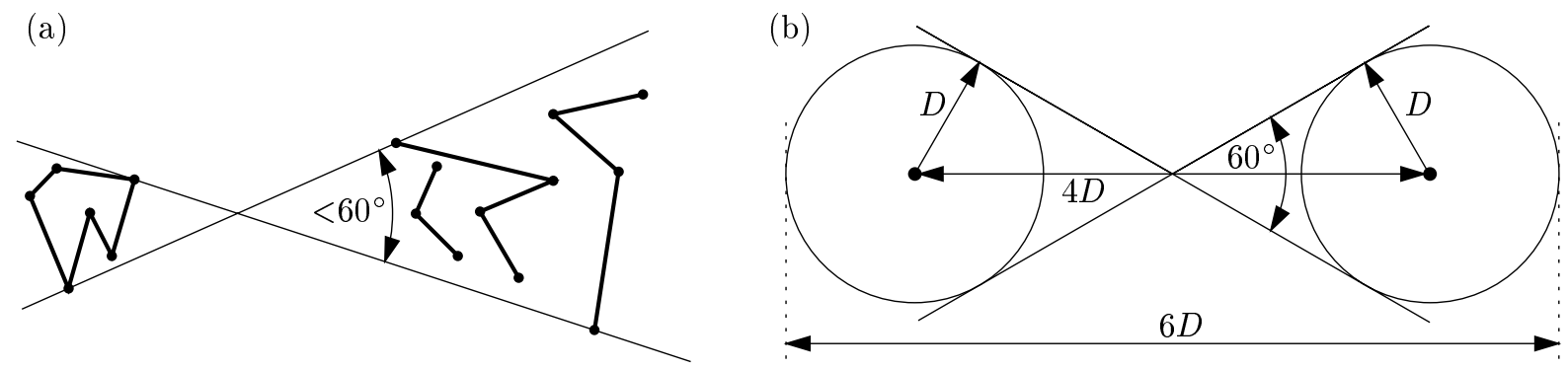

Figure 14: (a) Two well-separated clusters. (b) Inductive proof of Lemma 16. $D=D_{0} 6^{k-2}$ denotes the bound on the diameter of the two subclusters.

We now define a clustering procedure that will combine the components into clusters. (This procedure is similar to bottom-up hierarchical clustering [Eve93, HJ97], except that we pay no attention to the order in which we merge clusters.) We start with each component as a separate cluster. Whenever two clusters are not well-separated, we merge them into a single cluster. We repeat this until we end up with a single cluster or with a set of clusters that are pairwise well-separated (because otherwise we would perform another merge). The order in which we merge clusters has no effect on the outcome, because merging clusters may destroy well-separated pairs but never creates them, and hence only creates mergeable pairs and never destroys them. However, the clustering may change over time as the framework moves.

Lemma 16 A cluster with $k$ components has diameter at most $D_{0} 6^{k-1}$. 
Proof: By induction on $k$. Because a single component is connected, the case $k=1$ follows. A cluster with $k \geq 2$ components results from merging two clusters of at most $k-1$ components that are not well-separated. By induction, these two clusters are contained in two disks of radius at most $D_{0} 6^{k-2}$. If the distance between the disk centers were bigger than $4 \cdot D_{0} 6^{k-2}$, the two sets would be well-separated; see Figure 14(b). Hence the union of the two circles, which contains the cluster, has diameter at most $(2+4) \cdot D_{0} 6^{k-2}=D_{0} 6^{k-1}$.

So the quantity $D_{\max }=D_{0} 6^{n-1}$, which has already been introduced, is indeed an upper bound on the maximum possible distance between two points in the same cluster.

Lemma 17 A cluster with at least two components has width at least $m_{0} / 2$.

Proof: Assume for contradiction that a cluster with at least two components lies in a strip of width less than $m_{0} / 2$, which we can without loss of generality assume to be horizontal, as in Figure 15. Consider any two components in the cluster. By the definition of $m_{0}$, the distance between these two components is at least $m_{0}>m_{0} / 2$. Hence, there must be a vertical line separating the two components. Consider one of the two inner common tangents. The segment between the tangent points has length at least $m_{0}$ and lies in the strip. Therefore the angle between the tangent and the horizontal axis is less than $30^{\circ}$, and the same is true for the other tangent. It follows that any two components in the strip are well-separated, and therefore they do not belong to the same cluster.

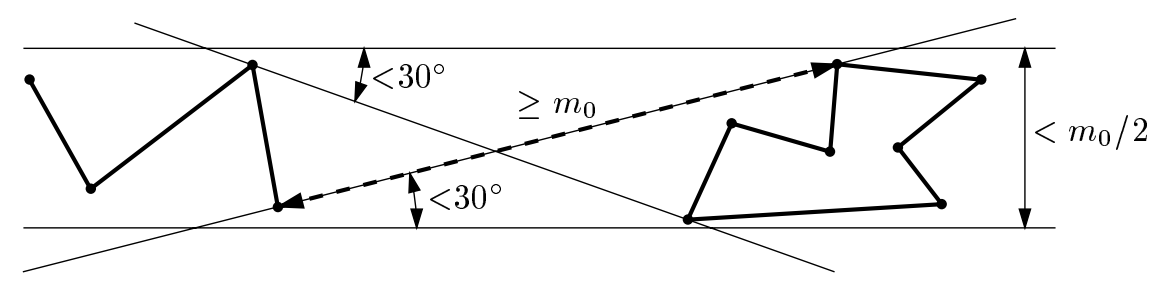

Figure 15: Proof of Lemma 17: two components in a thin strip.

Lemma 18 Let $\mathbf{p}_{1} \in V_{1}$ and $\mathbf{p}_{2} \in V_{2}$ be points in two well-separated clusters $V_{1}$ and $V_{2}$, and let $\overline{\mathbf{c}}^{(1)}$ and $\overline{\mathbf{c}}^{(2)}$ denote the center of gravity of the vertices in $V_{1}$ and $V_{2}$, respectively. Then the angle between the vectors $\mathbf{p}_{2}-\mathbf{p}_{1}$ and $\overline{\mathbf{c}}^{(2)}-\overline{\mathbf{c}}^{(1)}$ is at most $60^{\circ}$.

Proof: Immediate from the definition of well-separated, because the center of gravity of a cluster is within the convex hull of the cluster.

\section{A.4 Rounding up the proof}

Using Lemmas 14 and 15, we can bound all velocities $\mathbf{v}_{i}$ in terms of the sum of $\ell_{i j}^{\prime}$ over all pairs $\left(\mathbf{p}_{i}, \mathbf{p}_{j}\right)$ in the same cluster (plus some $\theta^{\prime}$ terms to accommodate single-component clusters). These quantities cannot grow indefinitely: we have an upper bound on the diameter of a cluster. 
Lemma 19 Let $V_{1}$ denote the set of vertices of a cluster and $T_{1} \subseteq V_{1}$ the set of degree-2 vertices, with angles $\theta_{j}$ for $j \in T_{1}, 0<\theta_{j}<\pi$. Let $\ell_{i j}$ denote the distance between vertices $i, j \in V_{1}$. Given an expansive motion of all vertices, let $\theta_{j}^{\prime}$ denote the derivative of $\theta_{j}$ with respect to time, $j \in T_{1}$, let $\ell_{i j}^{\prime}$ denote the derivative of $\ell_{i j}$ with respect to time, and let $\mathbf{v}_{j}$ denote the derivative of a vertex $\mathbf{p}_{j}$ with respect to time, $j \in V_{1}$. If the motion is pinned as in Lemma 14 (for the case of a single-component cluster) or Lemma 15 (for a multi-component cluster), then, for every $k \in V_{1}$,

$$
\left\|\mathbf{v}_{k}\right\| \leq C \cdot \sum_{i, j \in V_{1}} \ell_{i j}^{\prime}+D_{0} \cdot \sum_{i \in T_{1}} \theta_{i}^{\prime}
$$

where $C=5328 D_{\max }^{3} / m_{0}^{3}=8 \cdot 666 D_{\max }^{3} / m_{0}^{3}$.

Proof: The first term handles a cluster with at least two components by Lemma 15. We have width $w \geq m_{0} / 2$ by Lemma 17 and diameter $D \leq D_{\max }$ by Lemma 16 . The second term accounts for a single-component cluster and follows from Lemma 14.

Everything so far has been generic to expansive motions. Now we will use the precise form of $(7-9)$.

Lemma 20 For an arc-and-cycle set, let $T \subseteq V$ denote the set of degree-two vertices, and let $\theta_{j}$ denote the angle at vertex $j \in T, 0<\theta_{j}<\pi$. For a motion $\mathbf{p}_{j}(t)$ of this arc-and-cycle set, let $\ell_{i j}=\left\|\mathbf{p}_{i}(t)-\mathbf{p}_{j}(t)\right\|$ denote distances between vertices, let $D(t)=\max \left\{1, \max _{i, j \in V} \ell_{i j}(t)\right\}$ denote the diameter at time $t$ raised to be at least one, and let $\bar{\ell}_{i j}(t)=\min \left\{\ell_{i j}(t), D_{\max }\right\}$ denote the "truncated" distances. Then the solution $\mathbf{v}_{i}$ of the minimization problem (7-9) (defining a motion of the arc-and-cycle set) is bounded by

$$
\left\|\mathbf{v}_{k}\right\| \leq \frac{6 n}{m_{0} m_{1}}\left(C \sum_{i, j \in V} \bar{\ell}_{i j}^{\prime}+D_{0} \sum_{i \in T} \theta_{i}^{\prime}+2\right) \cdot D(t)
$$

Proof: The strategy of the proof is as follows:

1. Start with $\mathbf{v}_{i}$ given by the optimization problem (7-9).

2. In each cluster separately, "normalize" ("pin") $\mathbf{v}_{i}$ as indicated in Lemma 19, yielding "well-bounded" $\mathbf{v}_{i}$ 's for each cluster.

3. Combine these pinned motions into an overall expansive motion by adding a vector to each cluster that ensures that the clusters will fly away from each other. We have control over those vectors, and we guarantee that the overall motion is still well-bounded (bounded in terms of the sum of $\ell_{i j}^{\prime}$ over all pairs $i, j$ in the same cluster).

4. Thus we have shown the existence of a well-bounded solution. Using the minimizing property of the optimization problem we conclude that its solution is also well-bounded. 
Here are the details. Assume that there are $K$ clusters with vertex sets $V_{1}, \ldots, V_{K}$. We first consider the motion $\hat{\mathbf{v}}^{(r)}$ that is obtained from $\mathbf{v}$ by restricting it to a cluster $V_{r}$ and pinning it as in Lemma 14 or 15 . We know that

$$
\left\|\hat{\mathbf{v}}_{k}^{(r)}\right\| \leq \underbrace{C \cdot \sum_{i, j \in V_{r}} \bar{\ell}_{i j}^{\prime}+D_{0} \cdot \sum_{i \in T_{r}} \theta_{i}^{\prime}}_{\text {denoted by } H^{(r)}}
$$

for all $k \in V_{r}$.

By construction, all distance increases $\left\|\hat{\mathbf{v}}_{i}^{(r)}-\hat{\mathbf{v}}_{j}^{(r)}\right\|$ satisfy the conditions (8-9) for $i, j$ in the same cluster $V_{r}$. We will now add a vector to the motion of each cluster in order to ensure that the expansion (8) also holds for points in different clusters. Intuitively, we want the clusters to "fly apart," but in a controlled manner.

Let $\overline{\mathbf{c}}^{(r)}$ denote the center of gravity of cluster $r$. We set

$$
\overline{\mathbf{v}}_{k}:=2 \cdot \hat{\mathbf{v}}_{k}^{(r)}+E \cdot\left(\overline{\mathbf{c}}^{(r)}-\mathbf{p}_{1}\right)
$$

for $k \in V_{r}$, with an arbitrarily chosen fixed vertex $\mathbf{p}_{1}$ and where $E$ is defined by

$$
E=2\left(H^{(1)}+H^{(2)}+\cdots+H^{(K)}+2\right) / m_{0}>4 .
$$

Note that $m_{0} E / 2$ is the value in parentheses in the bound of Lemma 20 that we want to prove. For vertices in the same cluster, we get $\overline{\mathbf{v}}_{i}-\overline{\mathbf{v}}_{j}=2\left(\hat{\mathbf{v}}_{i}^{(r)}-\hat{\mathbf{v}}_{j}^{(r)}\right)$, and hence the constraint

$$
\left(\mathbf{v}_{i}-\mathbf{v}_{j}\right) \cdot\left(\mathbf{p}_{i}-\mathbf{p}_{j}\right) \geq\left\|\mathbf{p}_{i}-\mathbf{p}_{j}\right\|
$$

is "twice over-fulfilled":

$$
\left(\overline{\mathbf{v}}_{i}-\overline{\mathbf{v}}_{j}\right) \cdot\left(\mathbf{p}_{i}-\mathbf{p}_{j}\right) \geq 2 \cdot\left\|\mathbf{p}_{i}-\mathbf{p}_{j}\right\|
$$

So we get the penalty term

$$
\left[\left(\overline{\mathbf{v}}_{i}-\overline{\mathbf{v}}_{j}\right) \cdot\left(\mathbf{p}_{i}-\mathbf{p}_{j}\right)-\left\|\mathbf{p}_{i}-\mathbf{p}_{j}\right\|\right]^{-1} \leq\left[\left\|\mathbf{p}_{i}-\mathbf{p}_{j}\right\|\right]^{-1} \leq 1 / m_{1}
$$

We claim that this inequality also holds for points $i$ and $j$ in two different clusters $V_{r}$ and $V_{s}$. We have

$$
\begin{aligned}
\left(\overline{\mathbf{v}}_{i}-\overline{\mathbf{v}}_{j}\right) \cdot\left(\mathbf{p}_{i}-\mathbf{p}_{j}\right) & =\left(\hat{\mathbf{v}}_{i}^{(r)}-\hat{\mathbf{v}}_{j}^{(s)}\right) \cdot\left(\mathbf{p}_{i}-\mathbf{p}_{j}\right)+E\left(\overline{\mathbf{c}}^{(r)}-\overline{\mathbf{c}}^{(s)}\right) \cdot\left(\mathbf{p}_{i}-\mathbf{p}_{j}\right) \\
& \geq\left|E\left(\overline{\mathbf{c}}^{(r)}-\overline{\mathbf{c}}^{(s)}\right) \cdot\left(\mathbf{p}_{i}-\mathbf{p}_{j}\right)\right|-\left|\left(\hat{\mathbf{v}}_{i}^{(r)}-\hat{\mathbf{v}}_{j}^{(s)}\right) \cdot\left(\mathbf{p}_{i}-\mathbf{p}_{j}\right)\right| \\
& \geq E \cdot \underbrace{\frac{1}{2}\left\|\overline{\mathbf{c}}^{(r)}-\overline{\mathbf{c}}^{(s)}\right\| \cdot\left\|\mathbf{p}_{i}-\mathbf{p}_{j}\right\|}_{\text {by Lemma } 18}-\left(H^{(r)}+H^{(s)}\right) \cdot\left\|\mathbf{p}_{i}-\mathbf{p}_{j}\right\| \\
& \geq\left\|\mathbf{p}_{i}-\mathbf{p}_{j}\right\|\left[E m_{0} / 2-\left(H^{(r)}+H^{(s)}\right)\right] \\
& \geq 2 \cdot\left\|\mathbf{p}_{i}-\mathbf{p}_{j}\right\|
\end{aligned}
$$

by the definition of $E$. So $\overline{\mathbf{v}}$ is indeed a feasible solution to (8-9), and (27) holds for all struts $\{i, j\} \in S$. From $(25)$ and $\left\|\overline{\mathbf{c}}^{(r)}-\mathbf{p}_{1}\right\| \leq D(t)$ we obtain the bound

$$
\left\|\overline{\mathbf{v}}_{k}\right\| \leq 2 H^{(r)}+E \cdot D(t) \leq E m_{0}+E \cdot D(t) \leq 2 E \cdot D(t)
$$


4.48

44:09

44:10

44:11

44:12

44:13

44:14

44:15

44:16

44:17

44:18

44:19

44:20

44:21

44:22

$44: 23$

because we defined $D(t)$ to be at least 1 .

From this and from (27), the objective function (7) for the solution $\overline{\mathbf{v}}$ that we have constructed satisfies

$$
\begin{aligned}
& \sum_{i \in V}\left\|\overline{\mathbf{v}}_{i}\right\|^{2}+\sum_{\{i, j\} \in S}\left[\left(\overline{\mathbf{v}}_{i}-\overline{\mathbf{v}}_{j}\right) \cdot\left(\mathbf{p}_{i}-\mathbf{p}_{j}\right)-\left\|\mathbf{p}_{j}-\mathbf{p}_{i}\right\|\right]^{-1} \leq n \cdot(2 E \cdot D(t))^{2}+n^{2} / m_{1} \\
& \leq n^{2} \cdot(2 E \cdot D(t))^{2} \cdot \frac{1}{m_{1}}+\frac{n^{2}}{m_{1}} \cdot E^{2} \cdot D(t)^{2}=\frac{5 n^{2}}{m_{1}} \cdot E^{2} \cdot D(t)^{2} \leq\left(\frac{3 n}{m_{1}} \cdot E \cdot D(t)\right)^{2} .
\end{aligned}
$$

It follows that the optimal solution $\mathbf{v}$, from which we started, must also satisfy this inequality. Therefore we obtain,

$$
\sum_{i \in V}\left\|\mathbf{v}_{i}\right\|^{2} \leq \sum_{i \in V}\left\|\mathbf{v}_{i}\right\|^{2}+\sum_{\{i, j\} \in S}\left[\left(\mathbf{v}_{i}-\mathbf{v}_{j}\right) \cdot\left(\mathbf{p}_{i}-\mathbf{p}_{j}\right)-\left\|\mathbf{p}_{j}-\mathbf{p}_{i}\right\|\right]^{-1} \leq\left(\frac{3 n}{m_{1}} \cdot E \cdot D(t)\right)^{2}
$$

and hence, for every $k \in V$,

$$
\left\|\mathbf{v}_{k}\right\| \leq \sqrt{\sum\left\|\mathbf{v}_{i}\right\|^{2}} \leq \frac{3 n}{m_{1}} \cdot E \cdot D(t)
$$

Corollary $4 D(t) \leq D(0) e^{F(t)}$ where $F(t)=\frac{12 n}{m_{0} m_{1}}\left(C n^{2} D_{\max }+D_{0} n \pi+2 t\right)$.

Proof: By noting the relation $D^{\prime}(t) \leq 2 \max _{k \in V}\left\|\mathbf{v}_{k}\right\|$, we can apply the previous lemma to obtain an upper bound on $D^{\prime}(t) / D(t)=\frac{d}{d t} \log D(t)$ :

$$
\frac{D^{\prime}(t)}{D(t)} \leq \frac{6 n E}{m_{1}} \leq \frac{12 n}{m_{0} m_{1}}\left(C \sum_{i, j \in V} \bar{\ell}_{i j}^{\prime}+D_{0} \sum_{i \in T} \theta_{i}^{\prime}+2\right)
$$

Integrating and noting the bounds $\bar{\ell}_{i j} \leq D_{\max }$ and $\theta_{i} \leq \pi$, we obtain

$$
\log D(t)-\log D(0)=\int_{0}^{t} \frac{D^{\prime}(t)}{D(t)} d t \leq \frac{12 n}{m_{0} m_{1}}\left(C n^{2} D_{\max }+D_{0} n \pi+2 t\right) .
$$

We finally establish the result of the Boundedness Lemma (Lemma 10).

Corollary 5 The path length of a vertex $\mathbf{p}_{k}$ until time $T$ is bounded by

$$
\int_{t=0}^{T}\left\|\mathbf{v}_{k}\right\| d t \leq \frac{D(0) F(T)}{2} e^{F(T)}
$$

Proof: This follows from Lemma 20 and the previous corollary:

$$
\int_{0}^{T}\left\|\mathbf{v}_{k}(t)\right\| d t \leq \int_{0}^{T} \frac{3 n E}{m_{1}} D(t) d t \leq \int_{0}^{T} \frac{3 n E}{m_{1}} d t \cdot D(T) \leq \frac{F(T)}{2} \cdot D(0) \cdot e^{F(T)}
$$

Remark: By a different choice of shifting vectors in (26) instead of $E \cdot \overline{\mathbf{c}}^{(r)}$ for each cluster it is possible to avoid the exponential "blow-up" in the above bounds. 


\section{A.5 Proof of Lemma 15}

Unfortunately, the proof of Lemma 15 is quite elaborate. It is based on the special case of three points, which is elementary.

Lemma 21 Consider a triangle with sides $a, b, c$ bounded by $m \leq a, b, c \leq D$ and angles $\alpha, \beta, \gamma$ that are bounded by $\sin \alpha, \sin \beta, \sin \gamma \geq \mu>0$. If the sides of the triangle are expanded with velocities $a^{\prime}, b^{\prime}, c^{\prime} \geq 0$, then the change of the angles is bounded by

$$
\left|\alpha^{\prime}\right|,\left|\beta^{\prime}\right|,\left|\gamma^{\prime}\right| \leq \frac{2 D}{m^{2} \mu} \cdot\left(a^{\prime}+b^{\prime}+c^{\prime}\right)
$$

Proof: The cosine law gives $-\cos \alpha=-\left(b^{2}+c^{2}-a^{2}\right) /(2 b c)=\frac{1}{2}\left[a^{2} /(b c)-b / c-c / b\right]$. Taking the derivative and omitting negative terms on the right-hand side leads to

$$
\alpha^{\prime} \sin \alpha \leq \frac{1}{2}\left(\frac{2 a a^{\prime}}{b c}+\frac{b c^{\prime}}{c^{2}}+\frac{c b^{\prime}}{b^{2}}\right) \leq \frac{1}{2}\left(2 a^{\prime}+c^{\prime}+b^{\prime}\right) \frac{D}{m^{2}} \leq\left(a^{\prime}+c^{\prime}+b^{\prime}\right) \frac{D}{m^{2}}
$$

and thus, $\alpha^{\prime} \leq\left(a^{\prime}+b^{\prime}+c^{\prime}\right) \cdot D /\left(m^{2} \mu\right)$. We get the same upper bound for $\beta^{\prime}$ and $\gamma^{\prime}$. To obtain a lower bound, note that $\alpha+\beta+\gamma=\pi$ and hence $\alpha^{\prime}=-\beta^{\prime}-\gamma^{\prime} \geq-\left(a^{\prime}+b^{\prime}+c^{\prime}\right) \cdot 2 D /\left(m^{2} \mu\right)$. By symmetry, the same bound holds for the derivatives of the other two angles.

Proof (Lemma 15): Let $\mathbf{p}_{3}$ be the point whose distance $h$ from the segment $\mathbf{p}_{1} \mathbf{p}_{2}$ is largest. By assumption, $w / 2 \leq h \leq \sqrt{3 / 4} \cdot D$. So the points are contained in a rectangle $K$ of length $D$ and width $2 h$, see Figure 16 . Without loss of generality, we draw $\mathbf{p}_{1} \mathbf{p}_{2}$ horizontal from left to right and $\mathbf{p}_{3}$ above $\mathbf{p}_{1} \mathbf{p}_{2}$.

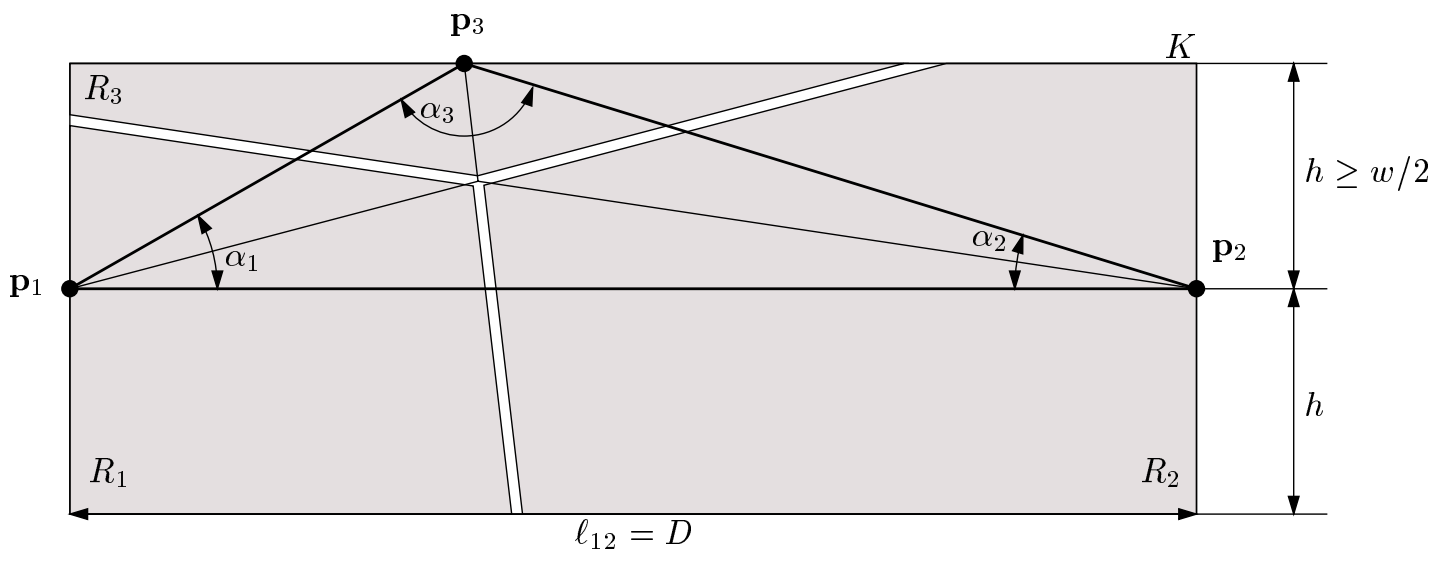

Figure 16: Proof of Lemma 15.

We use the triangle $\mathbf{p}_{1} \mathbf{p}_{2} \mathbf{p}_{3}$ with angles $\alpha_{1}, \alpha_{2}, \alpha_{3}$ as a "reference frame," and we measure the motion of the other points with respect to this frame. In particular, every point will be assigned to one of the sides of the reference triangle: We partition the rectangle $K$ into 


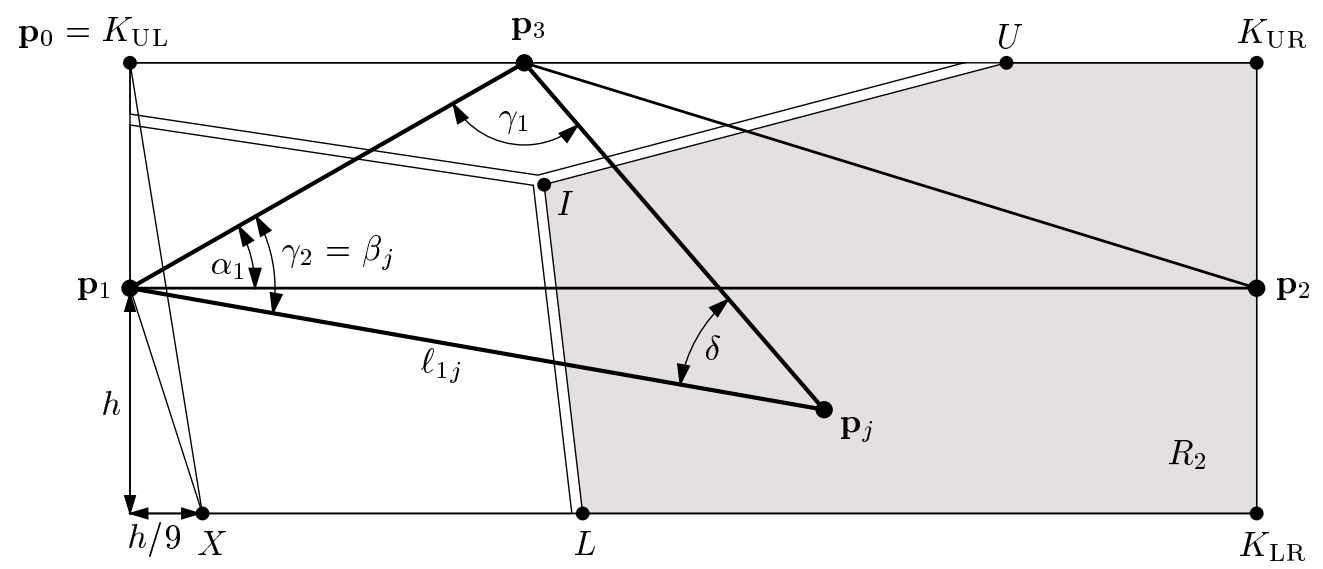

Figure 17: The triangle $\mathbf{p}_{3} \mathbf{p}_{1} \mathbf{p}_{j}$ associated with a point $\mathbf{p}_{j} \in R_{2}$ in the proof of Lemma 22 .

46:01

46:02

46:03

$46: 04$

46:05

$46: 06$

46:07

46:08

46:09

46:10

46:11

46:12

46:13

46:14

46:15

46:16

46:17

46:18

$46: 19$

46:20

46:21

46:22

three regions $R_{1}, R_{2}, R_{3}$ by extending the three angular bisectors from the incenter into the direction away from the vertices from which they emanate. $R_{i}$ is the region containing the point $\mathbf{p}_{i}$, for $i=1,2,3$. Now, every point $\mathbf{p}_{j}$ is assigned to the triangle side which lies opposite to the region in which it lies. For example, a point $\mathbf{p}_{j}$ in region $R_{1}$ is assigned to $\mathbf{p}_{2} \mathbf{p}_{3}$. We use this side as a basis of the triangle $\mathbf{p}_{2} \mathbf{p}_{3} \mathbf{p}_{j}$ that is associated with $\mathbf{p}_{j}$, and we select one of the angles at the basis as the reference angle $\beta_{j}$ for $\mathbf{p}_{j}$. If $\mathbf{p}_{j}$ lies in $R_{1}$, we select the angle at $\mathbf{p}_{2}$ in the triangle $\mathbf{p}_{2} \mathbf{p}_{3} \mathbf{p}_{j}$. If $\mathbf{p}_{j}$ lies in $R_{2}$, we take the angle at $\mathbf{p}_{1}$ in the triangle $\mathbf{p}_{3} \mathbf{p}_{1} \mathbf{p}_{j}$ as the reference angle $\beta_{j}$ for $\mathbf{p}_{j}$, see Figure 17 ; and for $\mathbf{p}_{j}$ in $R_{3}, \beta_{j}$ is the angle at $\mathbf{p}_{1}$ in the triangle $\mathbf{p}_{1} \mathbf{p}_{2} \mathbf{p}_{j}$. Note that these assignments are not completely symmetric. The rationale is to select a reference angle incident to $\mathbf{p}_{1}$ or $\mathbf{p}_{2}$.

The idea is that the triangle associated with each point cannot have arbitrarily small angles, and hence produces a "firm grip," tying the point to its base: The point cannot move an unlimited distance without increasing the pairwise distances $\ell_{i j}$ by a noticeable amount.

Lemma 22 The reference triangle $\mathbf{p}_{1} \mathbf{p}_{2} \mathbf{p}_{3}$ satisfies the assumptions of Lemma 21: The sines of the angles are at least $\mu:=w /(3 D)$ and the sides are at least $m:=w / 2$.

The triangle associated with each point satisfies the assumptions of Lemma 21: The sines of the angles are at least $\mu:=w /(20 D)$ and the sides are at least $m:=w / 4$.

This lemma is not difficult to see but the precise proof is a bit technical and it is given at the end. Lemmas 21 and 22 allow us to conclude:

$$
\left|\alpha_{1}^{\prime}\right|,\left|\alpha_{3}^{\prime}\right|,\left|\alpha_{3}^{\prime}\right| \leq \frac{2 D}{(w / 2)^{2} \cdot w /(3 D)} \cdot\left(\ell_{12}^{\prime}+\ell_{23}^{\prime}+\ell_{13}^{\prime}\right) \leq \frac{24 D^{2}}{w^{3}} \cdot L,
$$

with $L:=\sum_{i<j} \ell_{i j}^{\prime}$, and for every point $\mathbf{p}_{j}$ :

$$
\left|\beta_{j}^{\prime}\right| \leq \frac{2 D}{(w / 4)^{2} \cdot w /(20 D)} \cdot L \leq \frac{640 D^{2}}{w^{3}} \cdot L .
$$


Now we can bound the motion of $\mathbf{p}_{j}$ as follows. For $\mathbf{p}_{j} \in R_{3}$, the angle $\beta_{j}$ between the fixed ray $\mathbf{p}_{1} \mathbf{p}_{2}$ and the ray $\mathbf{p}_{1} \mathbf{p}_{j}$, and the length $\ell_{1 j}$ determine the position of $\mathbf{p}_{j}$ like polar coordinates. We therefore have

$$
\left\|\mathbf{p}_{j}^{\prime}\right\|=\sqrt{\left(\ell_{1 j} \beta_{j}^{\prime}\right)^{2}+\left(\ell_{1 j}^{\prime}\right)^{2}} \leq \ell_{1 j}\left|\beta_{j}^{\prime}\right|+\ell_{1 j}^{\prime}
$$

For $\mathbf{p}_{j} \in R_{2}$, the situation is similar, except that the angle between the " $x$-axis" $\mathbf{p}_{1} \mathbf{p}_{2}$ and $\mathbf{p}_{1} \mathbf{p}_{j}$ is given by $\alpha_{1}-\beta_{j}$, see Figure 17 :

$$
\left\|\mathbf{p}_{j}^{\prime}\right\| \leq \ell_{1 j}\left(\left|\alpha_{1}^{\prime}\right|+\left|\beta_{j}^{\prime}\right|\right)+\ell_{1 j}^{\prime}
$$

For $\mathbf{p}_{j} \in R_{1}$, the situation still more complicated, because the "origin" $\mathbf{p}_{2}$ of the coordinate system moves at a speed of $\ell_{12}^{\prime}$. The angle between the "negative $x$-axis" $\mathbf{p}_{2} \mathbf{p}_{1}$ and $\mathbf{p}_{2} \mathbf{p}_{j}$ is given by $\alpha_{2}-\beta_{j}$.

$$
\left\|\mathbf{p}_{j}^{\prime}\right\| \leq\left\|\mathbf{p}_{2}^{\prime}\right\|+\left\|\mathbf{p}_{l}^{\prime}-\mathbf{p}_{2}^{\prime}\right\| \leq \ell_{12}^{\prime}+\ell_{2 j}\left(\left|\alpha_{2}^{\prime}\right|+\left|\beta_{j}^{\prime}\right|\right)+\ell_{2 j}^{\prime}
$$

Putting everything together, we get for every $j$,

$$
\left\|\mathbf{v}_{j}\right\|=\left\|\mathbf{p}_{j}^{\prime}\right\| \leq L+D \cdot\left(\frac{24 D^{2}}{w^{3}} \cdot L+\frac{640 D^{2}}{w^{3}} \cdot L\right)+L=\left(2+\frac{664 D^{3}}{w^{3}}\right) \cdot L \leq \frac{666 D^{3}}{w^{3}} \cdot L,
$$

using $D \geq w$. This concludes the proof of Lemma 15 .

Finally, we have to prove Lemma 22. An elementary geometric fact is needed.

Lemma 23 In a triangle $A B C$ with height $h_{C}$ through $C$, the distance of $C$ from the angular bisector $g$ of the angle $\alpha$ at $A$ is at least $h_{C} / 2$.

Proof: Let $C^{\prime}$ be the foot of $h_{C}$, and let $D$ be the point on $g$ closest to $C$, see Figure 18 . Then $C^{\prime}$ and $D$ lie on the circle over the diameter $A C$. The chords $C D$ and $C^{\prime} D$ are equal since their peripheral angles at $A$ are both equal to $\alpha / 2$. (If $\alpha>\pi / 2$, the peripheral angle over $C^{\prime} D$ is not $\alpha / 2$ but $\pi-\alpha / 2$, and for $\alpha=\pi / 2$ the equality of the chords follows directly.) From the isosceles triangle $C C^{\prime} D$ we get $C D \geq h_{C} / 2$.

Proof (Lemma 22): We first prove the lower bound on the lengths of the edges. The basis edges $\mathbf{p}_{1} \mathbf{p}_{2}, \mathbf{p}_{2} \mathbf{p}_{3}$, and $\mathbf{p}_{3} \mathbf{p}_{1}$ all have lengths at least $h \geq w / 2$. By the construction of the three regions, the point $\mathbf{p}_{j}$ is separated from the point $\mathbf{p}_{i}$ on its base segment $(i=1,2$, or 3 ) by the angular bisector of the angle at $\mathbf{p}_{i}$ in the triangle $\mathbf{p}_{1} \mathbf{p}_{2} \mathbf{p}_{3}$. From Lemma 23 it follows that the distance $\mathbf{p}_{j} \mathbf{p}_{i}$ is at least $1 / 2$ times one of the three heights of the triangle $\mathbf{p}_{1} \mathbf{p}_{2} \mathbf{p}_{3}$. The smallest height of this triangle is opposite to the longest edge, which is $\mathbf{p}_{1} \mathbf{p}_{2}$. This height is $h$, and we obtain $\ell_{i j} \geq h / 2 \geq w / 4$.

Now let us consider the angles. Note that it suffices to check the condition of Lemma 21 for the smallest angle: if $\alpha$ is the smallest angle in a triangle with angles $\alpha, \beta, \gamma$, then $\alpha \leq \beta, \gamma \leq \pi-\alpha$, and hence $\sin \beta, \sin \gamma \geq \sin \alpha$.

The angle $\alpha_{1}$ in the triangle $\mathbf{p}_{1} \mathbf{p}_{2} \mathbf{p}_{3}$ is bigger than the angle between $\mathbf{p}_{1} \mathbf{p}_{2}$ and the ray from $\mathbf{p}_{1}$ to the upper right corner $K_{\mathrm{UR}}$ of the rectangle $K$ :

$$
\sin \alpha_{1} \geq \frac{h}{\sqrt{D^{2}+h^{2}}} \geq \frac{h}{\sqrt{2 D^{2}}} \geq \frac{w / 2}{\sqrt{2} \cdot D} \geq \frac{w}{3 D}
$$




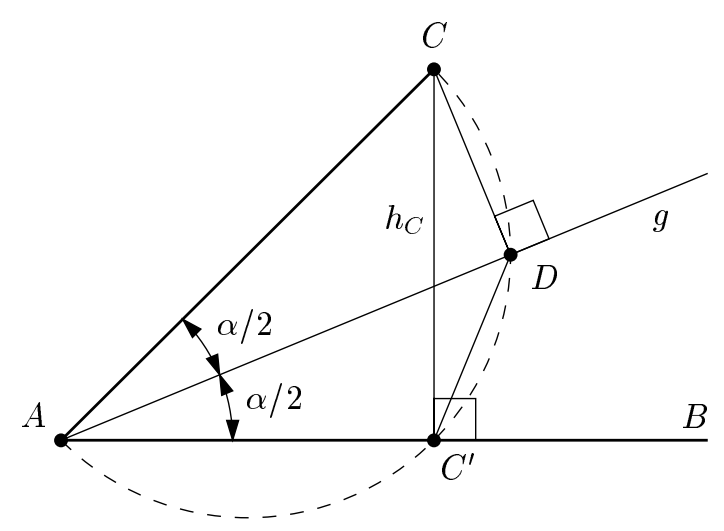

Figure 18: Proof of Lemma 23.

and similarly for $\alpha_{2}$. The angle $\alpha_{3}$ lies opposite to the longest edge of $\mathbf{p}_{1} \mathbf{p}_{2} \mathbf{p}_{3}$, and hence it is the largest angle. So we have shown the length and angle bounds for the reference triangle $\mathbf{p}_{1} \mathbf{p}_{2} \mathbf{p}_{3}$.

Let us now consider the triangle associated with a point $\mathbf{p}_{j}$. We denote the basis angles of the triangle by $\gamma_{1}$ and $\gamma_{2}$ and the angle at the apex $\mathbf{p}_{j}$ by $\delta$, see Figure 17. By construction of the angular bisectors, an angle $\gamma_{j}$ at the base is at least $1 / 2$ times the respective angle $\alpha_{i}$ in the reference triangle $\mathbf{p}_{1} \mathbf{p}_{2} \mathbf{p}_{3}$. So we have $\sin \gamma_{1}, \sin \gamma_{2} \geq \sin \frac{\alpha_{i}}{2} \geq\left(\sin \alpha_{i}\right) / 2 \geq w /(6 D)$.

So the only angle which remains to be checked is the third angle $\delta$ at $\mathbf{p}_{j}$. If $\mathbf{p}_{j}$ lies in $R_{3}$, $\delta$ lies opposite to the longest side of the triangle $\mathbf{p}_{1} \mathbf{p}_{2} \mathbf{p}_{j}$, and we are done.

In the remainder of the proof we show rather tediously that $\sin \delta \geq w /(20 D)$ when $\mathbf{p}_{j} \in R_{1}$ or $\mathbf{p}_{j} \in R_{2}$. Let us consider a triangle $\mathbf{p}_{3} \mathbf{p}_{1} \mathbf{p}_{j}$ with $\mathbf{p}_{j} \in R_{2}$, see Figure 17 . (The case $\mathbf{p}_{j} \in R_{1}$ is symmetric.) Keeping $\mathbf{p}_{j}$ and $\mathbf{p}_{1}$ fixed, $\delta$ is smallest when $\mathbf{p}_{3}$ moves on the upper side of the rectangle $K$ to the upper left corner $\mathbf{p}_{0}=K_{\mathrm{UL}}$. (This position of $\mathbf{p}_{3}$ is hypothetical because $\ell_{23}$ would be larger than $D$.) Keeping $\mathbf{p}_{1}$ and $\mathbf{p}_{0}$ fixed, the angle $\delta=\angle \mathbf{p}_{1} \mathbf{p} \mathbf{p}_{0}$ is a quasi-concave function of $\mathbf{p}=\mathbf{p}_{j}$ as $\mathbf{p}$ moves inside $K$. (This means that the region in $K$ where $\delta$ is bigger than some given threshold is alway convex.) Hence the minimum value of $\delta$ on any line segment occurs at one of its endpoints, and it suffices to check the lower bound for $\delta$ for the corners of the region $R_{2}$. More specifically, it is sufficient to check it for three points: (i) $\mathbf{p}=K_{\mathrm{UR}}$, the upper right corner of the rectangle $K$; (ii) $\mathbf{p}=K_{\mathrm{LR}}$, the lower right corner of $K$; and (iii) $\mathbf{p}=X$, a point on the lower edge of $K$ at distance $h / 9$ from the lower left corner.

The other corners of $R_{2}$ can be treated as follows: If $\mathbf{p}$ is the intersection $U$ of the bisector of $\alpha_{1}$ with the upper edge of $K$, then $\delta$ decreases as $\mathbf{p}$ moves right towards $K_{\mathrm{UR}}$. If the bisector of $\alpha_{1}$ intersects the boundary of $K$ in the right edge, then the intersection point lies on the segment between $K_{\mathrm{UR}}$ and $K_{\mathrm{LR}}$, and hence it is dominated by one of these points. (This situation occurs for region $R_{1}$ in Figure 17.) The incenter $I$ can be treated in the same way as $U$, by moving $\mathbf{p}$ horizontally to the right until it hits the right edge.

Another corner is the intersection $L$ of the bisector of $\alpha_{3}$ with the lower edge of $K$. We cover this case by showing that the point $X$ lies in region $R_{1}$. Therefore $L$ lies on the segment between $X$ and $K_{\mathrm{LR}}$, and hence it is dominated by these two points. If the bisector of $\alpha_{3}$ intersects the boundary of $K$ in the right edge, it is dominated by $K_{\mathrm{UR}}$ and $K_{\mathrm{LR}}$. The 
bisector cannot intersect the boundary of $K$ in the left edge because of the point $X$.

Let us now consider the points $\mathbf{p}=K_{\mathrm{UR}}$ and $\mathbf{p}=K_{\mathrm{LR}}$. By symmetry, $\mathbf{p}=K_{\mathrm{UR}}$ yields the same angle $\delta=\angle \mathbf{p}_{0} \mathbf{p p}_{1}$ as $\mathbf{p}=\mathbf{p}_{2}$ and that cannot be the minimum because it lies between $K_{\mathrm{UR}}$ and $K_{\mathrm{LR}}$. For $\mathbf{p}=K_{\mathrm{LR}}$, the sine law for the triangle $\mathbf{p}_{0} \mathbf{p p}_{1}$ with the angle $\delta=\angle \mathbf{p}_{0} \mathbf{p p}_{1}$ gives

$$
\frac{\sin \delta}{h}=\frac{\sin \angle \mathbf{p}_{0} \mathbf{p}_{1} \mathbf{p}}{\mathbf{p}_{0} \mathbf{p}}=\frac{D / \sqrt{D^{2}+h^{2}}}{\sqrt{D^{2}+(2 h)^{2}}} \geq \frac{D}{\sqrt{2 D^{2}} \cdot \sqrt{5 D^{2}}},
$$

using $h \leq D$. So we get

$$
\sin \delta \geq \frac{1}{\sqrt{10}} \cdot \frac{h}{D} \geq \frac{1}{2 \sqrt{10}} \cdot \frac{w}{D},
$$

and the lemma holds in this case.

Let us finally consider the point $X$. Since $h \leq \sqrt{3 / 4} \cdot D$, the point $X$ lies on the lower edge of $K$. We show that the distance of $X$ from the ray $\mathbf{p}_{3} \mathbf{p}_{1}$ is smaller than the distance from the ray $\mathbf{p}_{3} \mathbf{p}_{2}$, and therefore $X$ lies in $R_{1}$. The distance of $X$ from the ray $\mathbf{p}_{3} \mathbf{p}_{1}$ is at most the distance $X \mathbf{p}_{1}=\sqrt{1+1 / 81} \cdot h<1.0062 h$. The point on the ray $\mathbf{p}_{3} \mathbf{p}_{2}$ which is closest to $X$ lies either on the segment $\mathbf{p}_{3} \mathbf{p}_{2}$ or in the region to the right of the rectangle. In the latter case, the distance is at least $D-h / 9 \geq(\sqrt{4 / 3}-1 / 9) h>1.043 h$, which is larger than $X \mathbf{p}_{1}$. In the former case, the distance between $X$ and the segment $\mathbf{p}_{3} \mathbf{p}_{2}$ decreases if $\mathbf{p}_{3}$ or $\mathbf{p}_{2}$ are moved horizontally to the left. In the extreme cases, when $\mathbf{p}_{3}=K_{\mathrm{UL}}=\mathbf{p}_{0}$ and $\ell_{12}=D=\sqrt{4 / 3} \cdot h$, this distance can be calculated as $>1.439 h$, which is also larger than $X \mathbf{p}_{1}$. A final calculation show that the angle $\delta=\angle \mathbf{p}_{0} X \mathbf{p}_{1}$ satisfies $\sin \delta>0.055>1 / 20 \geq \frac{w}{20 D}$, since $w \leq D$, and the proof is complete. 